\title{
Les villages ferroviaires en Espagne : un mode de vie aux côtés du chemin de fer
}

Domingo Cuéllar Villar, Miguel Jiménez Vega et Francisco Polo Muriel Traducteur : Brigitte Ryffel

\section{(2) OpenEdition}

Édition électronique

URL : https://journals.openedition.org/rhcf/694

DOI : $10.4000 /$ rhcf.694

Éditeur

Rails \& histoire

Édition imprimée

Date de publication : 1 mai 2004

Pagination : $39-180$

ISSN : 0996-9403

Référence électronique

Domingo Cuéllar Villar, Miguel Jiménez Vega et Francisco Polo Muriel, « Les villages ferroviaires en Espagne : un mode de vie aux côtés du chemin de fer », Revue d'histoire des chemins de fer [En ligne], 31 | 2004, mis en ligne le 08 avril 2011, consulté le 22 avril 2022. URL : http://journals.openedition.org/ rhcf/694; DOI : https://doi.org/10.4000/rhcf.694 


\section{Domingo CUÉLLAR VILLAR, Miguel JIMÉNEZ VEGA FrancisCo POLO MURIEL}

\section{Les villages ferroviaires en Espagne : un mode de vie aux côtés du chemin de fer}

Traduit de l'espagnol par Brigitte Ryffel

\section{Introduction}

Les travaux de recherche sur l'histoire du chemin de fer en Espagne ont été abondants au cours des trente dernières années*. Comme nous le rappellent Miguel Muñoz et Javier Vidal' ${ }^{1}$, l'essor de l'historiographie moderne sur le chemin de fer remonte au début des années 1970, avec les travaux de Gabriel Tortella, Jordi Nadal et Miguel Artola.

Ces noms illustres ont été relayés au cours des décennies suivantes par les notables apports de Pedro Tedde, Antonio Gómez Mendoza, Rafael Anes, Pere Pascual ou José Morilla, pour ne citer que quelquesuns des plus significatifs. Les recherches qu'ils ont réalisées ont démontré l'importance du chemin de fer en Espagne depuis son apparition et ont alimenté le débat sur le degré de modernité apporté par ce moyen de transport à l'économie précaire espagnole, tout en tentant de quantifier ses bénéfices. Le point de vue économique et l'étude des grandes entreprises ferroviaires ont sans aucun doute contribué à la connaissance relativement précise de la naissance et du développement du chemin de fer en Espagne ${ }^{2}$. Toutefois, cet intérêt et cette richesse de la recherche sur l'histoire économique des chemins de fer contraste avec le faible nombre de travaux menés sur les aspects sociaux des mêmes compagnies ferroviaires. L'intérêt de ces derniers réside dans la connaissance qu'ils permettent des importantes transformations intervenues dans les domaines sociaux et professionnels au sein d'un secteur tel que les chemins de fer, qui a été le leader et la référence

* Ce texte est issu d'une communication présentée au III ${ }^{\mathrm{e}}$ Congrès d'histoire ferroviaire, tenu à Gijon (Asturies) du 23 au 26 septembre 2003.

1- Muñoz Rubio et Vidal Olivares (2001) (voir la bibliographie en fin d'article).

2- Dans ce contexte, deux ouvrages de référence récents sur l'étude du chemin de fer en Espagne peuvent être : Comín Comín, Martín Aceña, Muñoz Rubio et Vidal Olivares (1998) ; Muñoz Rubio, Sanz Fernández et Vidal Olivares (1999). 
principale du développement économique moderne pendant une grande partie du $\mathrm{xx}^{\mathrm{e}}$ siècle. Comme l'a montré Tortella dans ses premières recherches - ce que bien d'autres chercheurs ont ensuite confirmé le chemin de fer a accaparé une grande partie du mouvement des capitaux produits sur les marchés boursiers du moment ${ }^{3}$. Cela permit aux compagnies ferroviaires de maintenir leur leadership européen jusque dans les années 1950, aussi bien en termes de volume d'actifs financiers que de demande de main-d'œuvre et de création d'emplois ${ }^{4}$.

Les recherches ayant pour objet d'approfondir la connaissance du monde social du chemin de fer espagnol ont débuté en Espagne par les travaux d'Emerenciana Juez ${ }^{5}$. Cet auteur a centré ses études sur la transformation significative des relations sociales au sein des chemins de fer espagnols pendant la deuxième moitié du $\mathrm{XIX}^{\mathrm{e}}$ et les premières décennies du $\mathrm{xx}^{\mathrm{e}}$ siècle. Ce travail pionnier offre un panorama social des grandes transformations et des grands progrès, mais aussi des importantes limitations et des pénuries qui allaient s'accentuer dans les années 1910, époque où sont apparues de grandes transformations sociales dans le monde ferroviaire : journée de travail de huit heures, augmentations de salaires, reconnaissance de droits sociaux, etc.

Plus récemment, Tomás Martínez Vara et Esmeralda Ballesteros ont approfondi ces aspects et ont effectué une première quantification de l'évolution de la main-d'œuvre employée par le secteur ferroviaire au cours de la période d'exploitation des anciennes compagnies, en particulier de la Compañía de los Caminos de Hierro del Norte de España (NORTE) et de la Compañía del Ferrocarril de Madrid a Zaragoza y Alicante (MZA) $)^{6}$ Leurs apports nous permettent de confirmer l'importance du chemin de fer en tant qu'activité économique de main-d'œuvre (labor intensive), à des périodes de faible mécanisation, entraînant une importante dispersion de la main-d'œuvre dans les différents sites établis par le chemin de fer sur tout le territoire espagnol.

C'est dans ce contexte que naissent les premiers foyers de population purement ferroviaires, naturellement situés aux carrefours des lignes, ou autour des grands ateliers de réparation et des dépôts de locomotives. Il faut en outre avoir à l'esprit que, dans des agglomérations existantes, qu'il s'agisse de petits villages ou de grandes villes, les colonies

3- Tortella Casares (1973), Pascual Domènech (1999).

4- Carreras et Tafunell (1993).

5- Juez Gonzalo (1992) et (2000).

6- Ballesteros Doncel et Martínez Vara (2001). 
de personnel ferroviaire se multiplient autour des gares et des sites principaux de production ferroviaire. Ces nouveaux foyers de population ne sont donc pas les seuls endroits où le personnel ferroviaire s'est établi. Les environs des gares ferroviaires ont été l'un des premiers espaces à être occupés par les cheminots, jusqu'à générer au cours des ans de véritables quartiers propres à la corporation. Ces quartiers, en principe de nature spontanée, se sont vu renforcés à la fois par l'élan du coopératisme ferroviaire, qui a promu la construction de logements après la promulgation en 1911 de la loi sur les habitations à prix modéré (Ley de Casas Baratas), et par l'initiative des entreprises du secteur ellesmêmes qui étendirent également les limites de ces quartiers, à travers la construction de nouveaux blocs de logements. Mis à part ce type d'habitat, concentré et typique des centres urbains déjà consolidés, il convient de distinguer celui qui s'est peu à peu disséminé le long de la voie ferrée, pour déployer de véritables légions de travailleurs qui, accompagnés de leurs familles respectives, avaient pour mission de surveiller les installations fixes.

L'objet de cet article est ainsi mis en évidence : l'étude et la connaissance des différents types de villages ferroviaires apparus en Espagne ex novo depuis la moitié du XIX $x^{\mathrm{e}}$ jusqu'à la fin du $\mathrm{Xx}^{\mathrm{e}}$ siècle, période de crise pour ces localités qui verront diminuer de façon alarmante leur population à la suite des profondes transformations technico-productives intervenues dans l'exploitation ferroviaire à partir des années 1980.

Ce travail est ainsi divisé en trois parties. La première propose une définition et replace dans leur contexte les villages ferroviaires et leurs différents sous-types ou variantes, en fonction de leur importance et de la dotation en équipements dont ils disposaient au moment de leur plus grande activité. On y procède également à une interprétation régionale de ce phénomène, qui n'a pas eu un impact identique dans toutes les zones du pays, ni la même intensité dans toutes les compagnies ferroviaires. La seconde partie entre dans le vif du sujet, en analysant les différentes formes et cas particuliers des « espaces physiques » de ces foyers de population. Ce point analyse les différentes typologies des « structures urbaines » de ces sites qui, dans tous les cas, ont connu un développement relativement anarchique et ont dû nécessairement s'adapter à la disponibilité du sol non utilisé par les compagnies ferroviaires pour leur exploitation. L'emplacement des logements et leur expansion n'étaient pas toujours les plus adéquats, dans la mesure où ils étaient fortement pénalisés par la proximité des voies ferroviaires et des autres espaces de travail liés au chemin de fer, qui produisaient 
des nuisances, étaient source d'inconfort et de dangers significatifs pour leurs habitants. La dernière partie analyse en détail les différents aspects du monde social au sein des villages ferroviaires. On y aborde l'important problème du logement, certes partagé avec les autres citoyens, mais qui, dans le cas ferroviaire, comporte d'importantes singularités qu'il convient de relever. Dans le cadre de l'analyse des différents services communs que l'on trouvait au sein de ces agglomérations, cette partie analyse également le rôle joué par l'éducation des enfants, qu'il s'agisse de l'enseignement officiel ou de celui dit «non réglementé», qui inculquait aux jeunes apprentis l'esprit de la profession ferroviaire. On y souligne également l'importance de la religion, profondément ancrée dans la société espagnole, et qui - tout particulièrement à partir de 1940 contribua pour le domaine ferroviaire aux processus de catéchisation fort courants à cette époque en Espagne. Le rôle des services médicaux et des différentes structures administratives de ces localités complète cette vision générale.

Malgré l'importance des points jusqu'ici indiqués, nous pouvons affirmer que les travaux sur ces aspects sont absents de l'historiographie ferroviaire espagnole 7 . Pour cette raison, il semble tout particulièrement intéressant de présenter ici les sources qui ont servi de référence dans cette recherche en cours d'exécution, dont une sélection est présentée dans la liste proposée en fin d'article.

Nous pouvons distinguer deux types de sources : celles générées par les entreprises et qui sont parvenues jusqu'à nos jours soit sous forme de dossiers administratifs déposés dans des archives, soit sous forme de publication officielle, comme les rapports des différents conseils d'administration, les règlements et instructions pour le personnel et les articles et informations qui apparaissent dans les magazines d'entreprises. Elles constituent un premier corpus d'informations, assez fourni, qui permet de connaitre tous les aspects de l'activité qui, en matière sociale, a été développée par les entreprises privées puis par les entreprises publiques qui ont succédé aux précédentes après les processus de nationalisation mis en œuvre avant et après 1941. Ces outils permettent en outre d'atteindre un double objectif : connaittre, comme

\footnotetext{
7- Ce n'est pas ce qui s'est produit dans le domaine international, où nous trouvons des travaux intéressants, Drummond (1990), Strangleman (2002) et Taksa (2002). Pour l'Espagne, jusqu'à ce jour, nous disposons des travaux suivants : Inventario de los Poblados Ferroviarios en España (inventaire des villages ferroviaires en Espagne), Fundación de los Ferrocarriles Españoles et Instituto del Patrimonio Histórico Español, 2003 (inédit) et Cuéllar Villar, Jiménez Vega, De Lus Roldán et Polo Muriel (2003).
} 
nous le disions auparavant, le rôle joué par les entreprises à cet égard et les restrictions et devoirs qui incombaient aux employés en échange des bénéfices sociaux qu'ils recevaient (logement, éducation, assistance médicale, etc.). Ce premier groupe de sources permet également, dans certains cas, de connaitre de première main les besoins du personnel ferroviaire quant à ces avantages sociaux. L'un des plus criants pour l'ensemble de la collectivité était celui du logement, en raison de ses faibles dimensions (qui dans bien des cas empêchaient des familles de taille moyenne d'y vivre confortablement) et de l'absence de commodités élémentaires (eau et électricité, principalement). Cette information, qui est normalement associée à des demandes formulées par les intéressés pour agrandir leurs logements ou y effectuer des travaux de réhabilitation, constitue une documentation très utile, bien que rare et biaisée, qui permet de connaître les aléas de la vie domestique familiale et des conditions matérielles de son logement. N'oublions pas de citer l'information fournie par les magazines ferroviaires publiés sous les auspices des entreprises ou la publicité générée dans le secteur. Celles-ci fournissent d'importants documents qui mettent l'accent sur les moments de loisirs et de jeu les plus importants de ces endroits, comme par exemple les fêtes religieuses ou profanes qui marquaient le rythme des journées dans ces foyers de travail et de cohabitation ouvrière.

L'autre groupe important de sources est constitué par les travaux réalisés sur le personnel ferroviaire ou en rapport avec lui, qu'il s'agisse de thèses doctorales menées au cours des deux dernières décennies ${ }^{8}$ ou de projets de recherche engagés ces dernières années qui ont commencé à porter leurs fruits. Cependant, ces magnifiques travaux se nourrissent des sources primaires qui ont été sommairement décrites dans le paragraphe précédent, ainsi que des témoignages de personnel ferroviaire interviewé par les historiens spécialistes de ce type de méthodologie et de l'usage des sources orales'. Ce dernier type de source, faute de récits autobiographiques ${ }^{10}$, se révèle suffisamment éclairant pour découvrir certains aspects essentiels susceptibles d'expliquer l'amalgame entre la vie familiale et la vie professionnelle dans les villages ferroviaires.

8- Juez Gonzalo (1992) et Tomás García (1991).

9- Folguera (sous la dir. de), Díaz Sánchez, Domínguez Prats et Gago Sánchez (2002) et Sanz Hernández (2000). À propos de l'utilisation des sources orales pour la reconstruction d'aspects de la vie professionnelle ferroviaire, nous recommandons la lecture de l'article de Strangleman (2002).

10- En Espagne, il existe jusqu'à ce jour peu d'autobiographies de cheminots qui aient été publiées : Zurdo Olivares (1912), Tejada Herrero et Zavala (1999), et Castro Iglesias (1999). 
En ce sens, à partir du projet développé par la Fundación de los Ferrocarriles Españoles sur l'histoire des villages ferroviaires, il a été procédé au recueil de témoignages oraux qui, à l'avenir, seront à la disposition des chercheurs intéressés par leur consultation ${ }^{11}$.

\section{Villages ferroviaires : définition, emplacements et développement}

Comme nous l'avons déjà souligné dans un travail précédent, nous entendons par village ferroviaire:

«les foyers de population qui sont nés ex novo d'un lien étroit avec l'arrivée du chemin de fer, qui ont été majoritairement peuplés par des employés des différentes compagnies exploitantes et qui, en règle générale, étaient situés dans des lieux d'importante activité ferroviaire (embranchements de lignes ou nœuds ferroviaires, gares de triage, terminus de ligne, etc.), et complétaient ainsi les installations qui s'y trouvaient (gares de triage, ateliers, dépôts et remises de locomotives, etc. $)^{12}$.

Néanmoins, tous les foyers de population ferroviaires n'ont pas une origine identique, pas plus que leur importance n'est équivalente, et bien évidemment leur développement présente des caractéristiques différentes. Une première division fait une distinction entre villages, hameaux et quartiers. À leur tour, chacun de ces groupes présente une séparation entre population purement ferroviaire et population mixte, pour faire une distinction entre ceux qui avaient une activité presque exclusivement ferroviaire et ceux où le chemin de fer partageait son rôle avec d'autres activités économiques, comme les mines, l'agriculture ou tout autre secteur industriel. Parmi les trois groupes principaux village, hameau ou quartier - l'attribution du terme de village correspondrait aux foyers de population naissant d'une nouvelle création, très étroitement liés au commencement de l'activité ferroviaire. Ils étaient ainsi majoritairement peuplés par les membres du personnel ferroviaire et leurs familles et se trouvaient généralement dans un environnement inhabité. Cette circonstance, et la présence d'habitants de plus en plus nombreux dans le village, rendaient nécessaire l'apparition de services complémentaires qui permettaient de rendre la localité habitable. Ils disposaient ainsi d'écoles, financées par l'entreprise ferroviaire elle-même ou en association avec l'État. On y érigeait également une église pour

11- De fait, nous offrons plus bas quelques extraits de ces témoignages à titre documentaire.

12- Cuéllar Villar, Jiménez Vega, De Lus Roldán et Polo Muriel (2003). 
assurer les offices religieux avec une régularité certaine. On disposait également d'un dispensaire qui permettait d'assurer un service de santé, quelquefois supérieur à celui dont disposaient les localités environnantes. Un autre élément important était l'existence d'un économat, géré par l'entreprise elle-même ou cédé à des tiers et qui, dans certains cas, était ambulant. Ce caractère de « village ferroviaire pur», nous l'avons jusqu'à présent identifié pour un total de quatorze localités : Algodor (Madrid), Almorchón (Badajoz), Arroyo-Malpartida (Cáceres), Bobadilla (Málaga), Chinchilla (Albacete), La Encina (Alicante), Espeluy (Jaén), Gare LinaresBaeza (Jaén), Fuente del Arco (Badajoz), Maçanet-Massanas (Gérone), Monfragüe (Cáceres), Moreda (Grenade), Puente de los Fierros (Asturies) et Vadollano (Jaén). Le groupe des «villages ferroviaires mixtes » est constitué par Barruelo de Santullán (Palencia), CanfrancGare (Huesca), Castejón de Ebro (Navarre), Ojos Negros (Teruel), Port-Bou (Gérone), Los Rosales (Séville), Surroca (Gérone), Valencia de Alcántara-Gare (Cáceres), Venta de Baños (Palencia) et Villanueva del Río y Minas (Séville) (fig. 1).

Les hameaux étaient des foyers de population plus modestes, également habités en majorité par du personnel ferroviaire et dans lesquels ont été implantés quelques services élémentaires pour garantir la vie quotidienne de la communauté qui s'y installait, mais ce n'a pas été le cas partout : leur mode de vie était alors relativement précaire et dépendait des relations avec l'extérieur. Dans le groupe des hameaux ferroviaires purs, nous trouverions ainsi Calasparra (Murcie), Doña María-Ocaña y Nacimiento (Almería) et Roda de Bará (Tarragone). Tandis que les hameaux ferroviaires mixtes seraient Agramón (Albacete), Aljucén (Badajoz), Mengíbar-Las Palomeras (Jaén) et Puerto de La Laja (Huelva).

Le troisième groupe défini est celui des quartiers ferroviaires, qui étaient des foyers de population physiquement intégrés dans d'autres agglomérations plus importantes qui existaient déjà, mais qui possédaient une forte identité ferroviaire et se trouvaient habituellement à la périphérie d'une ancienne agglomération, ou très proche de celle-ci. Dans la catégorie des quartiers ferroviaires de caractère pur, signalons les cas de La Almozara (Saragosse), Astorga-San Andrés (León), Belmez (Cordoue), Mora la Nova (Tarragone), Los Prados (Málaga), Torralba (Soria) et Vicálvaro (Madrid). Parmi les quartiers ferroviaires mixtes, citons : Aldea Moret (Cáceres), Guadix (Grenade), Las Matas (Madrid), Sagunto-Puerto (Valence), San Vicent de Calders (Tarragone), Santa Fe-Alhama y Serón (Almería) et Villabona de Asturias (Asturies). 
Après avoir fait référence aux enclaves ferroviaires sur la base de leur fonctionnalité, la figure 1 nous permet d'établir de premières conclusions quant à la typologie de leur localisation géographique dans le contexte national. En premier lieu, une plus forte présence de ce type de foyer de population est indéniable dans la moitié sud de la Péninsule, en particulier en Andalousie, en Estrémadure, à Madrid et dans le Levant espagnol. Un total de 31 des 47 foyers de population inclus dans cette étude est situé dans ces régions. Une première explication de la plus grande concentration dans ces zones pourrait être recherchée dans l'existence de vastes espaces inhabités, à travers lesquels fut réalisé le tracé du chemin de fer pour relier des points extrêmes de l'espace espagnol. Les cas de Chinchilla, La Encina, Monfragüe, Almorchón ou Moreda illustrent sans aucun doute parfaitement une telle circonstance. Dans ce cas, il s'agit de villages ferroviaires purs qui, de même que les hameaux ferroviaires purs, se sont créés ex novo dans des zones inhabitées à travers lesquelles passait le chemin de fer, principalement au niveau de nœuds ou de liaisons ferroviaires, ou, dans le cas des hameaux, auprès d'installations ferroviaires telles que des dépôts ou ateliers.

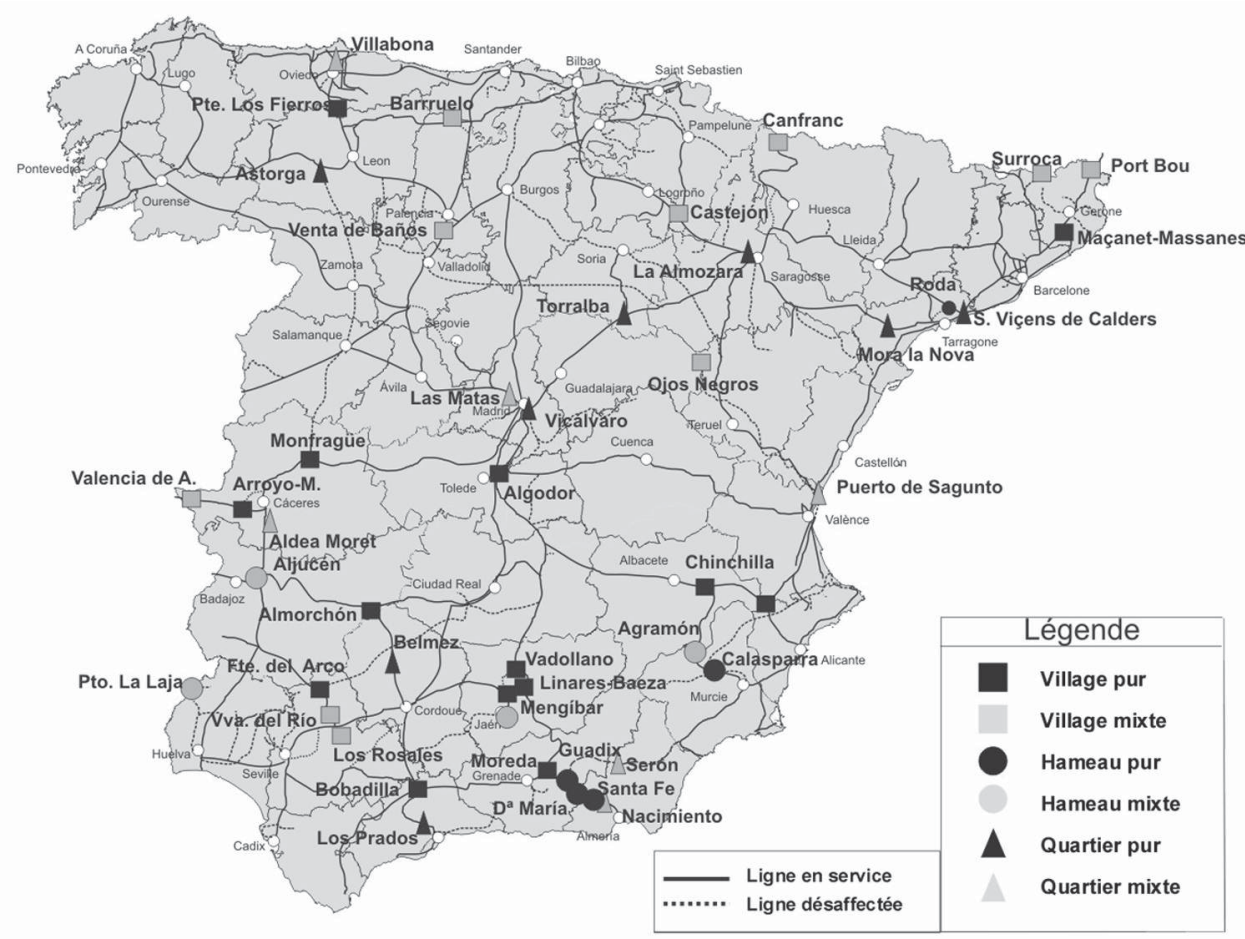

Figure 1. Les villages ferroviaires en Espagne. Classification par villages, hameaux et quartiers. Source : élaboré par les auteurs. 
Une seconde évaluation consiste à connaittre l'emplacement et la distribution des foyers de population à caractère mixte, c'est-à-dire ceux qui possédaient d'autres activités économiques en dehors du chemin de fer. Nous pouvons en citer deux types : les sites miniers-ferroviaires et les sites frontaliers. Les premiers correspondraient aux cas de Barruelo de Santullán, Ojos Negros, Surroca et Villanueva del Río y Minas. Pour ce qui est des sites ferroviaires frontaliers, citons Canfranc-Gare, PortBou et Valencia de Alcántara-Gare.

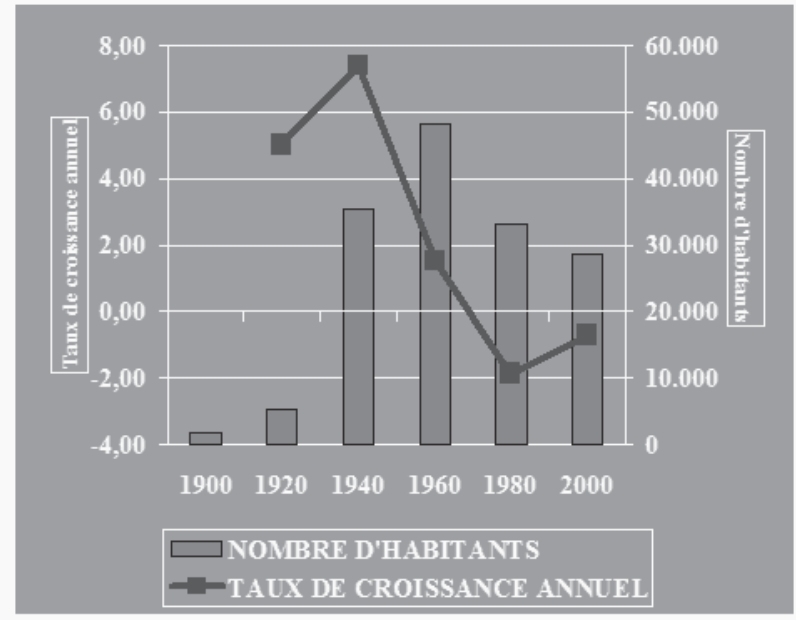

Figure 2. Évolution de la population dans les villages ferroviaires. Source : élaboré par les auteurs à partir de données de l'Institut espagnol de statistiques (INE) pour plusieurs années.

Mis à part les caractéristiques communes des foyers de population ferroviaires et leur distribution géographique, il est intéressant d'apporter ici une première évaluation quantitative du nombre de leurs habitants et de leur comportement au cours du temps. Comme nous pouvons le vérifier par la figure 2, la croissance de la population ferroviaire fut constante depuis le début du $\mathrm{XIX}^{\mathrm{e}}$ siècle, comme le reflètent les indicateurs de population de l'Institut espagnol de statistiques (INE) qui rassemblent, non sans aléas, les données de la population dans ces foyers de population, en particulier pour ce qui concerne les villages et hameaux; cela est plus difficile à suivre dans le cas des quartiers, dans la mesure où les données apparaissent quelquefois mêlées à la population principale. Malgré ces problèmes, la tendance de l'évolution de la population est parfaitement marquée, avec la phase de croissance constante à laquelle nous faisions allusion plus haut, pour s'arrêter dans les années 1980 qui marquent le 
début de leur dépeuplement. Ainsi, la population comptabilisée en 1960 dans notre échantillon était de 48159 habitants, alors qu'en 2000 elle était descendue à 28713 . De plus, si nous tenons compte du fait que cinq agglomérations (Castejón de Ebro, Las Matas, Los Rosales, Venta de Baños et Villanueva del Río y Minas) accaparaient $75 \%$ de ces habitants, nous pouvons nous faire une idée de l'ordre de grandeur de ce dépeuplement $t^{13}$. Parmi les villages qui ont perdu le plus grand pourcentage de population au cours de cette période se trouvent Arroyo-Malpartida (perte de 96,7\%), Almorchón (93,6 \%), Moreda (93,3\%), Monfragüe $(92,4 \%)$, Algodor (92\%), Vadollano (91,1\%), Chinchilla (89\%), La Encina $(83,3 \%)$ et Guadix $(65,2 \%)$. Il s'agit précisément de ceux qui, au moment de leur apogée, montraient la plus grande identité ferroviaire.

\section{Les espaces physiques : développement et distribution}

Une gare et plusieurs groupes de maisons dispersées autour du faisceau de voies : il s'agit là de l'une des formes les plus simples, et les plus justes, de définition formelle des poblados ferroviaires. Face aux exemples espagnols les plus élaborés de villages ouvriers (les villages de colonisation agricole $)^{14}$ ou aux cas moins harmonieux mais montrant une certaine volonté de rationaliser l'espace urbain (par exemple, les colonies textiles catalanes) ${ }^{15}$, les villages ferroviaires suscitent chez le visiteur, dans un premier temps, la sensation d'un certain chaos urbain.

Ce qui ne veut pas dire, bien au contraire, que les logements ainsi agencés sans forme préalable ne répondaient pas à des normes de qualité : celles-ci, compte tenu des années au cours desquelles ils furent érigés, étaient bien supérieures à celles de leur entourage. Plus encore, la trame urbaine sans forme de leurs voiries et édifices n’était pas forcément pire que celle des villages agricoles situés dans leurs alentours : elle était même bien supérieure (largeur des voies, présence d'espaces ouverts, alignements montrant une certaine cohérence...). On peut même dire davantage : la qualité des villages ferroviaires dépasse largement les exemples réputés les plus déplorables des foyers de population industriels, comme les villages miniers ${ }^{16}$. En effet, le paternalisme du patron textile catalan, l'État bienfaiteur des villages agricoles ou l'entrepreneur ferroviaire étaient très éloignés de l'entrepreneur concessionnaire de mines, spéculateur

13- Quarante ans plus tôt, au moment de plus fort peuplement, ces cinq villages comptabilisaient à eux seuls $47 \%$ des habitants de villages, hameaux et quartiers ferroviaires (données de l'Institut espagnol de statistiques - INE).

14- Villanueva Paredes et Leal Maldonado (1990).

15- Dorel Ferre (2003).

16- González Urruela (2001). 
qui, tout particulièrement au XIX ${ }^{\mathrm{e}}$ siècle, n'attachait généralement aucune importance au confort de ses travailleurs pour maximiser à tout prix les bénéfices (bien évidemment, il existe de très rares exceptions, comme dans le cas de la localité asturienne de Bustiello ${ }^{17}$ ), sans prendre en considération les conséquences négatives de cette tactique qui étaient précisément contrecarrées par la construction de villages industriels.

En marge de ces comparaisons, et sans entrer dans l'appréciation des qualités intrinsèques des logements des villages ferroviaires espagnols, à part quelques rares exceptions, on observe de façon quasi générale l'absence d'une volonté de planification dans la création de ces quartiers ouvriers. La courte vision urbanistique de l'entrepreneur ferroviaire, les grands intervalles de temps écoulés entre les phases de création de ces espaces (genèse créée par des impulsions décousues au cours - en moyenne - de presque quarante années) et les contraintes spatiales existantes (d'ordre topographique et surtout l'existence des lignes de chemin de fer elles-mêmes) sont la cause de cette mosaïque urbaine atypique présente sur une bonne partie du territoire péninsulaire.

Mis à part quelques exceptions internes montrant une certaine cohérence intrinsèque (petits quartiers suivant des normes standard de conception urbaine), les villages étaient des espaces de cohabitation sans pratiquement aucune articulation interne, traversés par des voies ferroviaires, sans zones symboliques de référence, autour desquelles gravitait la dynamique sociale. Nous tenterons cependant de donner l'image la plus claire possible les différents cas particuliers que l'on peut trouver.

\section{Les formes urbaines}

Même s'il n'existe aucun modèle urbain assimilable à celui des villages ferroviaires, il est certain que les habitations tendaient à se regrouper, presque toujours dans une position très proche des voies. Les rails et les bâtiments d'exploitation sont des éléments communs à tous les villages et c'est dans ce cadre caractéristique constant que nous avons défini un certain nombre de modèles. Bien que la topographie joue un rôle dans la disposition des rues et des pâtés de maisons, l'agencement du faisceau des voies, du bâtiment voyageurs, des quais, des aiguillages, du dépôt, etc., et des parcelles contiguës étaient essentiels dans la conception de ces quartiers si particuliers. Dans quelques rares cas, pour des raisons largement expliquées (Canfranc ${ }^{18}$, Belmez, Ojos $\left.\operatorname{Negros}^{19} \ldots\right)$, nous pouvons rencontrer des logements relativement

17- Ayuntamiento de Mieres (1999).

18- Parra De Mas (1988).

19- Sanz Hernández (2000). 
éloignés des voies. La classification que nous présentons ci-dessous et son développement postérieur dans le texte peuvent servir de référence ouverte au débat (fig. 3).

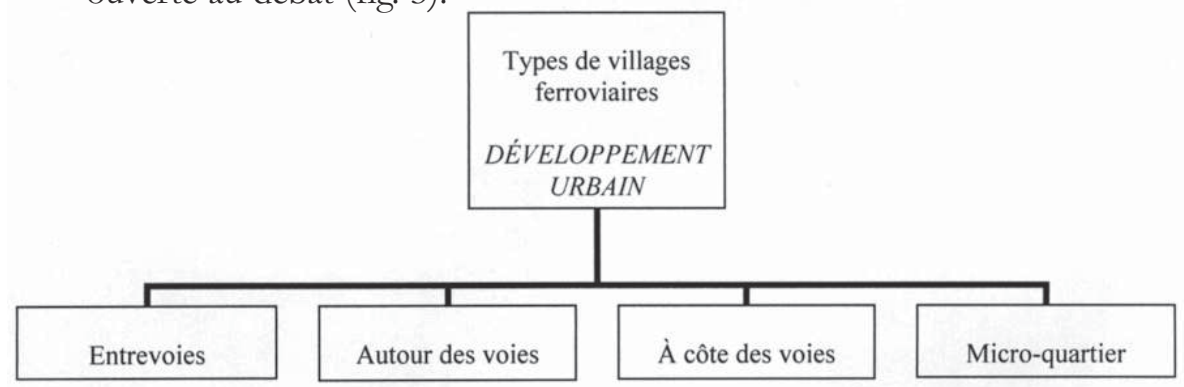

Figure 3. Classification des poblados ferroviaires en fonction de leur trame urbaine. Source : élaboré par les auteurs.

\section{Entrevoies}

Il existe quelques exemples, bien que peu fréquents, de groupes de logements situés sur le faisceau de voies d'une gare. La plupart des cas correspondaient habituellement à des nœuds ferroviaires, où l'on profitait des espaces laissés libres dans les zones qui séparaient les lignes ferroviaires, sur les faisceaux de sortie de la gare, pour construire des logements ouvriers.

Il s'agit de cas certainement précaires, confinés entre les rails, dont l'un des plus significatifs est le quartier ferroviaire de Torralba ${ }^{20}$ dans la province de Soria (situé à la bifurcation des voies vers Soria et Saragosse). Compte tenu de sa relative modernité (années 1950), les accès étaient bien résolus par des passages inférieurs, ce qui n'était pas le cas du quartier minier ferroviaire d'Aldea Moret ${ }^{21}$. Sa plus grande ancienneté et sa composante minière ont sans doute entraîné une certaine précarité dans ce quartier d'entrevoie de Caceres. Espeluy, à Jaén, est un autre cas typique, où cohabitaient même les logements d'entreprises différentes (Compañía de los Ferrocarriles Andaluces - ci-après ANDALUCES - et MZA). Ici, contrairement à la disposition triangulaire des foyers de population précédents, les logements occupaient une longue et étroite frange entre un long tronçon où les voies des deux compagnies s'étendaient parallèlement, jusqu’à leur séparation entre Linares et Alcázar d'une part et Puente Genil et Séville d'autre part.

20- Jiménez Vega (1992).

21- Gómez Amelia (1978). 


\section{Autour des voies}

Après la typologie des villages «enfermés », passons au deuxième grand groupe de typologie urbaine. Il s'agirait du cas contraire, où les logements « enfermaient» les voies des enceintes des gares.

Ce ne sont pas non plus des cas correspondant à un modèle dans lequel prime la fonctionnalité interne du noyau urbain. Disposer des maisons et des équipements de part et d'autre d'un faisceau de voies avec des trains en circulation constitue un environnement des moins enviables pour tout urbaniste et citoyen «normal». Le cas le plus évident de cette typologie serait le village madrilène d'Algodor ${ }^{22}$, où cette distribution était nuancée par un agencement des logements en fonction de leur proximité du lieu où le cheminot remplissait son emploi : dépôt de traction, tour d'aiguillage ou bâtiment voyageurs. Il n'y avait pas de passages réglementés entre les deux zones de logements, et les voies étaient posées à quelques mètres des bâtiments (fig. 4).

En marge de ce cas typique existent d'autres exemples qui montrent plusieurs nuances. Bobadilla, à Málaga, pourrait correspondre à la catégorie suivante, mais comme nous l'avons souligné plus haut, la croissance " par impulsions successives » des agglomérations conduit la Red Nacional de Ferrocarriles Españoles (RENFE), dans les années 1950, à construire un ensemble de 80 logements du côté opposé à la zone urbaine déjà consolidée, ce qui brisait un schéma répondant jusqu'alors à une certaine harmonie. Le cas de la gare de Fuente de Arco (Badajoz) n'en est pas moins particulier. De même qu'à Espeluy, il y avait à la fois des voies de la Ferrocarriles de Vía Estrecha (FEVE), titulaire à l'époque du chemin de fer qui montait jusqu'à Puertollano, et de la RENFE, pour la ligne Mérida-Los Rosales. Les compagnies construisirent leurs maisons de part et d'autre des deux gares en vis-àvis, en répartissant proportionnellement les édifices habituellement communs : église et école. À Serón (Almería) ${ }^{23}$, les logements des cheminots étaient alignés d'un côté du faisceau de voies, mais le quartier des employés de la mine, surgi à proximité de la gare, se développa de façon à envelopper le tracé de la ligne Guadix-Almendricos. Dans des proportions bien supérieures, ce processus s'est aussi enclenché à Villanueva del Río y Minas (Séville) ${ }^{24}$, où une grande agglomération a fini par envelopper une voie qui serpentait entre plusieurs puits de mine.

22- Jiménez Vega et Polo Muriel (1999).

23- Reche Sánchez (1991).

24- Tomás García (1991). 

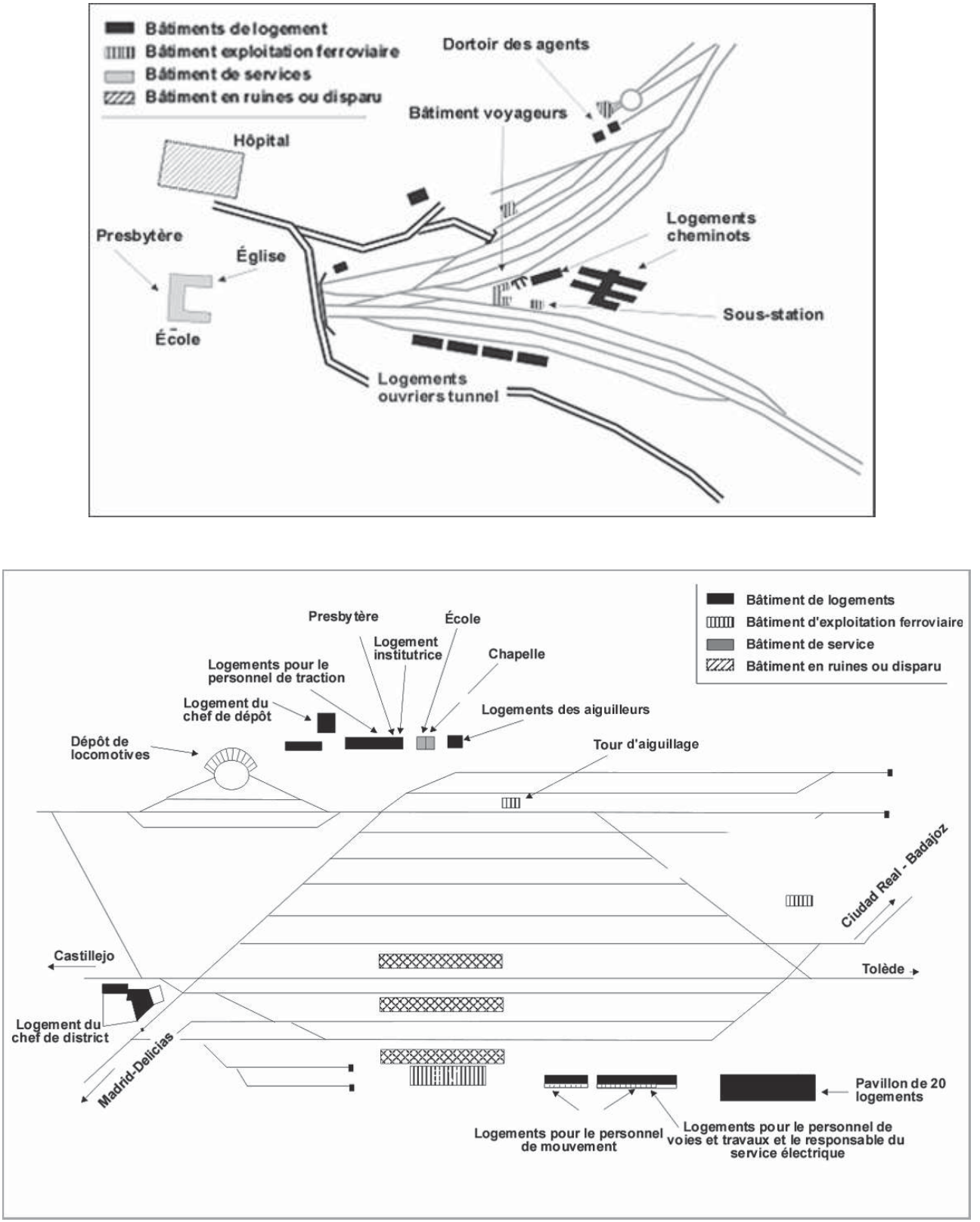

Figure 4 : Exemples de disposition de bâtiments dans des villages du type " entrevoies" et " autour des voies": de haut en bas, Torralba (Soria) et Algodor (Madrid). Source : élaboré par les auteurs. 
Les rails et puits de mines ont généré un espace désarticulé où le seul indice de planification apparaissait dans le quartier où étaient logés les cadres des mines et où se trouvaient les centres scolaires.

\section{À côté des voies}

Peut-être s'agit-il de la typologie où la contrainte ferroviaire était la moins décisive, c'est pourquoi les voies et bâtiments d'exploitation n'y constituent pas des contraintes cruciales du développement urbain du village. Cependant, il est vrai que les logements étaient généralement distribués très près des sites purement ferroviaires : cette contrainte existait donc, bien que dans une moindre mesure que dans les cas précédents. Ainsi, le mode de vie interne du village ne se différencierait pas beaucoup de ce qui pourrait s'observer dans des localités voisines sans rapport avec la dynamique ferroviaire, et la distribution des lieux de sociabilité serait relativement semblable.

Notre volonté systématique nous a conduits à considérer, dans cette catégorie, un certain nombre de sous-divisions répondant à des cas particuliers d'un certain intérêt. Nous aurions ainsi ce que nous appelons les "villages adossés ». Dans ce cas, les logements étaient alignés en épis, à proximité de la zone des voies, sans pratiquement aucun développement en profondeur. Tel est le cas d'Astorga-San Andrés (le plus parfait), La Almozara, Los Prados et même LinaresBaeza (dans sa première phase de développement). Dans certains cas, ces villages adossés avaient, sur certains de leurs tronçons (rappelons leur grand développement longitudinal), quelques expansions qui créaient des places ou des rues transversales.

Ce serait, par exemple, le cas des grands villages d'Estrémadure, Arroyo-Malpartida ${ }^{25}$ ou Monfragüe (fig. 5). Le cas d'Almorchón (Badajoz) évoque également cette étroite frange de logements parallèle aux voies, même si, dans ce cas, s'ajoute le fait que l'alignement ne se situait pas sur le bord des voies mais légèrement à l'écart du faisceau principal, pour créer une sorte de village à l'apparence un peu plus autonome que d'autres ici présentés. Une situation semblable correspond au village madrilène de Las Matas, où les logements s'agencent sous une forme longitudinale, mais en marge des voies, qu'il s'agisse de celles de circulation générale de la ligne Madrid-Irun ou de celles du faisceau de triage responsable de leur existence.

25- Domínguez Pedrera (1993). 


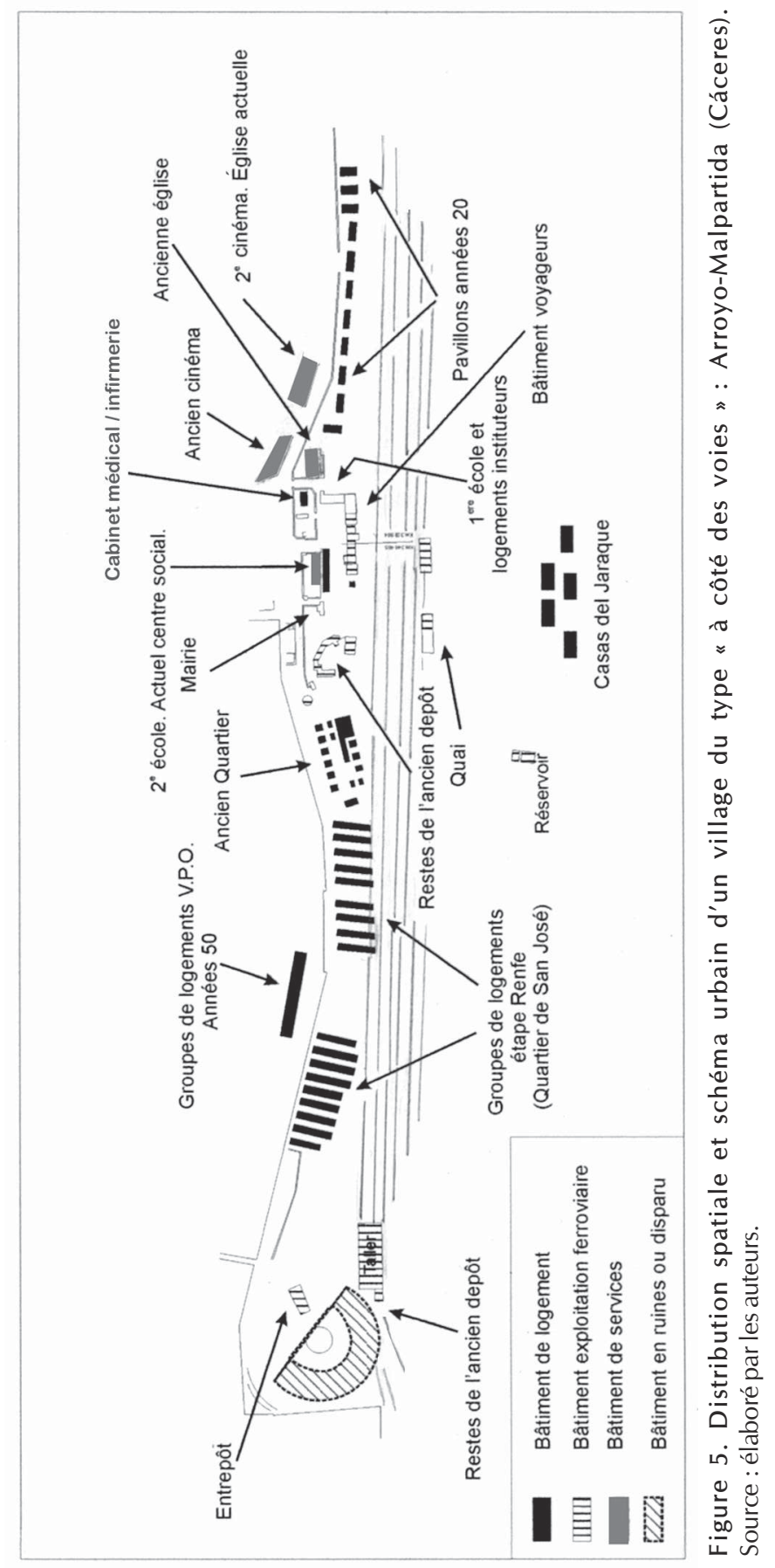




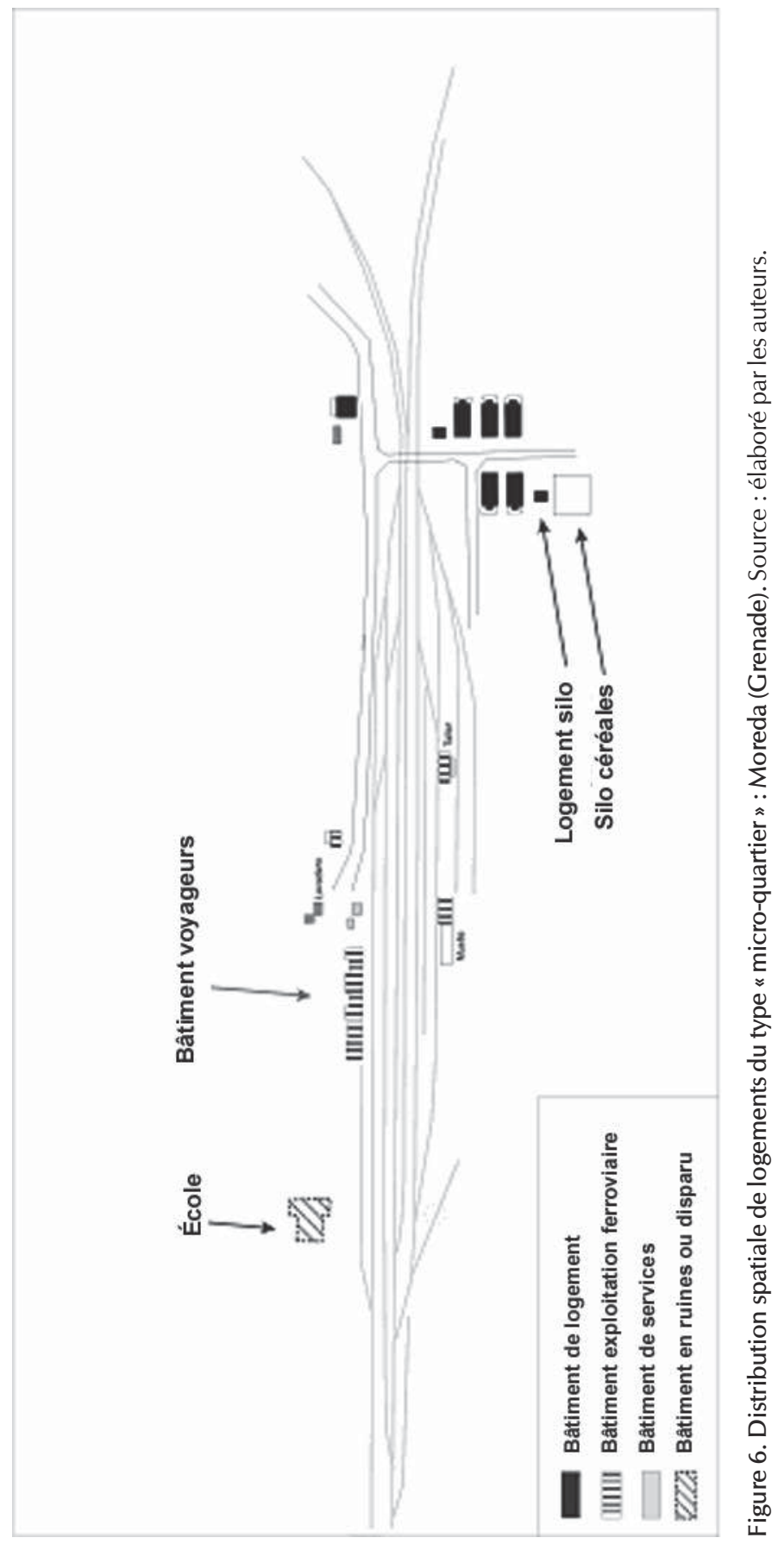




\section{Micro-quartiers}

Cette catégorie prétend rassembler un cas de figure en quelque sorte intermédiaire, avec une plus grande pureté de style interne, plus distinct que dans les cas précédents des zones d'exploitation. Ce serait par exemple le cas du quartier de Vicálvaro ${ }^{26}$, ensemble harmonieux ayant subsisté à côté des ateliers et des dépôts de l'ancien chemin de fer du Tajuña. Nous y placerions également le petit groupe de logements de Maçanet, situé à côté des voies mais à l'écart de l'ensemble des bâtiments d'exploitation de la gare, les maisons de Moreda (bien que certains bâtiments de services soient situés à proximité de la gare) (fig. 6) ou le hameau rural de Doña María-Ocaña, dans la région d'Almería.

\section{L'organisation formelle}

Comme on l'a indiqué, les villages ferroviaires ont pour caractéristique pratiquement générale l'absence d'une structure urbaine conventionnelle. C'est-à-dire que, si on les compare avec une localité rurale quelconque, on ne retrouve pas la structure habituelle de la place où sont rassemblés le pouvoir temporel (mairie) et spirituel (l'église), ensemble autour duquel, en fonction des contraintes topographiques existantes, les logements et autres installations (école, marché, lavoir...) sont disposés. Cette circonstance, bien évidemment, n’est jamais apparue dans les cas des hameaux ferroviaires, puisque ceux-ci ne disposaient d'aucun bâtiment collectif. Une donnée curieuse, si on les compare avec les localités agricoles ou textiles, c'est que les églises des villages ferroviaires ne connurent jamais le développement qu'elles eurent dans ces autres cas, dans la mesure où les entreprises ferroviaires érigèrent des chapelles. On peut noter que pratiquement aucune chapelle de village n'avait de clocher (exception faite de Port-Bou), alors que celui-ci était le référentiel principal du paysage des villages agricoles.

Là où l'on observe en revanche des exemples d'espaces jouissant d'un certain niveau d'organisation formelle, c'est dans les localités les plus développées, dont le cas le plus démonstratif est celui de Las $\mathrm{Matas}^{27}$. Nous avons déjà parlé de Las Matas comme exemple de "village adossé ». De disposition allongée, il présente à une extrémité l'agora où se trouvent l'église, les écoles et le dispensaire. La rue centrale aligne les logements, schéma que la RENFE a maintenu lorsque, dans le cadre de deux nouvelles «impulsions » constructives, dans les années 1950 et 1970, elle développa d'autres modèles architecturaux qui

26- Jiménez Vega et Polo Muriel (2003a).

27- Sánchez Domingo (1993). 
prolongeaient le schéma urbain déjà abordé dans les années 1920. Le cas d'Arroyo-Malpartida est moins harmonieux, mais on y apprécie ce centre où sont rassemblés l'église, l'école, le dispensaire, et même un cinéma. Ce groupe d'immeubles était fermé par le bâtiment voyageurs de la gare, centre névralgique de l'exploitation ferroviaire qui, dans une certaine mesure, voyait son rôle central renforcé par cette présence physique au sein du « cœur » vivant de l'agglomération.

Dans le village de La Encina, à Alicante, l'église joue un rôle directeur dans le paysage urbain, un tant soit peu désarticulé par le mélange de lotissements de plusieurs entreprises ferroviaires $\left(\mathrm{AVT}^{28}\right.$, NORTE, MZA et RENFE) et de logements construits par des particuliers. Situé face au bâtiment voyageurs (de l'autre côté des voies), face au temple, s'étend l'ensemble des voies, avec un parc parallèle au faisceau (à une cote plus élevée, presque comme un mirador) qui recrée un espace de place centrale dans la localité.

La Encina est un exemple clair de mélange de constructions de l'entreprise et de logements particuliers, où le modèle urbain se brise encore plus et ressemble aux zones de logements des villages espagnols, dépourvus de toute tentative de planification.

\section{Les espaces de cohabitation et de travail : conditions de vie, relations sociales et organisation administrative}

Travail, loisirs et vie familiale dans les villages ferroviaires

Les vies professionnelle, sociale et familiale dans un village ferroviaire se trouvaient étroitement liées entre elles. C'était l'endroit où se déroulait une grande partie des journées de travail des cheminots qui y étaient employés, et où vivaient leurs familles. Le village ferroviaire rassemblait des éléments typiques de l'exploitation ferroviaire et offrait également tous les services essentiels au fonctionnement social d'un noyau urbain. Tous ces facteurs, à savoir ceux d'ordre productif et ceux rendant possible la vie quotidienne des habitants, étaient dans la plupart des cas apportés par les entreprises qui exploitaient la ligne ferroviaire au sein de laquelle s'insérait le village.

Comme nous l'avons déjà précisé, les villages ferroviaires surgirent normalement dans des zones auparavant dépeuplées, où les différentes entreprises choisirent de situer des embranchements, dépôts de matériel et traction, ateliers ou gares de triage. Le fonctionnement de ces sites

28- Compañía de los Ferrocarriles de Almansa a Valencia y Tarragona. 
ferroviaires requit, jusqu'à la moitié des années 1970, un grand volume de ressources humaines qui, en tout état de cause, était bien supérieur à celles qui se trouvaient dans d'autres gares situées dans d'importantes agglomérations, comme les capitales de provinces ou les chefs-lieux de cantons. Ainsi, des agglomérations ferroviaires telles que ArroyoMalpartida, Plasencia-Empalme (par la suite Monfragüe) ou Baeza-Empalme (Gare Linares-Baeza) comptaient sur un nombre d'employés supérieur à Cáceres, Plasencia ou Jaén.

Ces îlots ferroviaires reproduisaient dans la classification des travailleurs rattachés à leurs divers établissements la structure d'exploitation habituelle du service ferroviaire. D'une part, les agents chargés du contrôle de la circulation et de la composition et de la surveillance des trains (service du Mouvement). Un autre groupe habituellement nombreux était constitué par les travailleurs du service de la Traction, chargés de la conduite des locomotives. Le service du Matériel rassemblait les agents chargés de la maintenance et de l'entretien du matériel roulant. Les brigades de Voie et Travaux avaient pour mission de réaliser les tâches de maintenance et d'entretien de la voie, ainsi que des bâtiments et dépendances de la gare et du village. Parmi les autres services qui recevaient une importante dotation d'agents dans ces sites ferroviaires, citons : Éclairage, Électrification, Gardes jurés (Gardería Jurada), Service médical, personnel de l'Économat, etc. Cette maind'œuvre concentrée, mais caractérisée par une forte division du travail, se déplaçait fréquemment au cours de sa journée de travail vers d'autres points de la ligne et des gares proches pour des tâches d'entretien, réparation et maintenance. À la fin de leur journée de travail, qui était organisée normalement selon un système de roulements qui couvraient 24 heures sur 24, les agents revenaient à leur lieu de résidence habituel, ce qui permettait ainsi la fixation des familles en un endroit déterminé et représentait pour les entreprises un véritable problème, dans la mesure où il était nécessaire de loger et de fournir tous les types d'équipements et services élémentaires permettant d'améliorer le niveau de vie de la communauté ferroviaire. C'est ainsi que surgissent les demandes en matière d'éducation, de santé, de culte et d'articles de première nécessité. Nous mènerons plus bas une analyse plus détaillée de tout ce qui a été signalé jusqu'à présent, qui nous permettra d'envisager la reconstitution de l'évolution historique de ces nouveaux villages et la connaissance des facteurs d'identité de groupe et de cohésion sociale qui leur sont propres. 


\section{Le logement}

C'est probablement le logement qui constituait le principal problème des habitants des villages ferroviaires (fig. 7). La plupart des villages ferroviaires qui se sont créés en Espagne autour de ces centres de travail n'ont pas été correctement planifiés par les entreprises, contrairement par exemple au cas de la Compagnie du Nord en France ${ }^{29}$ ou à d'autres secteurs productifs (industrie textile catalane, villages de colonisation agricole.... $)^{30}$. En Espagne, les entreprises tentèrent de résoudre le problème au fur et à mesure qu'il se présentait à travers les demandes formulées par les travailleurs pour accéder aux logements de la compagnie, ou des réclamations des syndicats ferroviaires ou encore par l'intermédiaire des services médicaux des compagnies ferroviaires eux-mêmes, qui alertaient la direction sur les conséquences pour la santé de l'exiguité de l'espace dans lequel vivaient les familles des employés ${ }^{31}$. Ce grave problème fut toujours résolu de façon partielle par les entreprises, dans la mesure où il existait toujours des employés et des familles qui devaient chercher à se loger dans les localités avoisinantes ou résider dans les wagons où ils effectuaient les trajets ${ }^{32}$, construire des logements précaires ou aménager des grottes dans les parois des collines proches de la gare ${ }^{33}$.

29- Voir la chronique journalistique signée G.V. portant le titre « Une leçon d'urbanisme » et publiée dans Le Temps du 23 juin 1923. Disponible à l'Archivo Histórico Ferroviario (AHF) : S-0235-97. Ce bref article offre une information précieuse sur les cités jardins promues par la Compagnie du Nord pour ses employés. Elle mentionne concrètement trois exemples de ce type de villes : Tergnier, Lille-Délivrance et Lens.

30- Villanueva Paredes et Leal Maldonado (1990), Serra I Rotés (2000) et Clua I Mercadal (2001).

31- Les dossiers conservés au sein des AHF sur les villages ferroviaires dépendant de MZA (Almorchón, La Encina, Algodor, Los Rosales, Baeza, Chinchilla, Espeluy, etc.) et de ANDALUCES (Bobadilla, Moreda, etc.) permettent de découvrir de nombreux cas de demandes de construction de logements ou d'agrandissement de ceux existant, déposées par les employés, ainsi que les rapports élaborés par les services médicaux sur les conditions matérielles des logements et leur surpeuplement. Les demandes des syndicats ferroviaires en matière de logement sont rassemblées dans la thèse de Juez Gonzalo (1992), p. 393-396.

32- Dans les témoignages oraux que nous avons recueillis, il est fréquemment fait mention de l'hébergement provisoire de familles de cheminots dans des wagons fermés.

33- À l'instar des cas du quartier ferroviaire de Guadix (Grenade) et du hameau ferroviaire de Santa Fé de Alhama (Almería), entre autres nombreux points. 


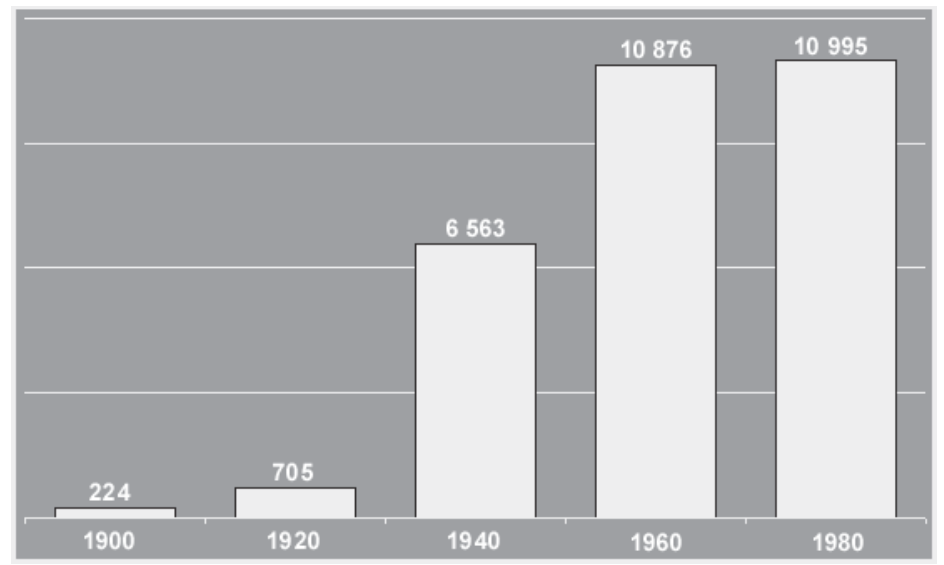

Figure 7. Évolution du parc de logements dans les villages ferroviaires, 1900-1980. Source : élaboré par les auteurs à partir de données de l'Institut espagnol de statistiques (INE) pour plusieurs années.

L'entreprise MZA, consciente du problème, élabora dans les années 1911 et 1921 deux rapports dans lesquels elle parvenait à quantifier le besoin de logements dans les gares de son «ancien réseau ». Le rapport de 1921, qui est le plus complet des deux, estimait à 1083 les logements qu'il était nécessaire de construire, exception faite de ceux qui étaient localisés dans le domaine du Réseau catalan ${ }^{34}$. Malgré ce déficit de bâtiments, en 1920 MZA fournissait un logement gratuit à 6481 employés et 567 mineurs de La Reunión jouissaient de maisons à loyer réduit ${ }^{35}$. Ce problème devint encore plus aigu après la mise en œuvre progressive de la journée de huit heures pour la plupart des agents dans les années 1920. Cela signifie que les dotations en personnel de presque tous les services ont eu tendance à augmenter, pour finalement accroître la population résidant dans les villages ferroviaires ${ }^{36}$. Pour faire face à cette évolution, les compagnies ont dû envisager la

34- AHF, C-1305-05.

35- Voir Memoria de la Compañia MZA de l'année 1920, p. 18.

36- Le groupe de recherche de la Fundación de los Ferrocarriles Españoles sur l'histoire des villages ferroviaires en Espagne a quantifié que la population résidente dans les villages, hameaux et quartiers ferroviaires identifiés lors de la réalisation de l'Inventaire des villages ferroviaires est passée de 5272 en 1920 à 35479 en 1940. Source INE: Nomenclátores de población. 
construction de logements dans certains de ces foyers de population, comme le précise le mémoire rédigé à l'appui du projet d'extension de la gare d'Astorga Empalme, appartenant à la Compañía del Ferrocarril de Madrid, Cáceres y Portugal y Oeste de España (MCP) ${ }^{37}$. La plupart de ces travaux étaient pris en charge par l'État, à travers des fonds de la Caja Ferroviaria qui permirent la modernisation des installations et du matérie ${ }^{38}$.

Mis à part le rôle joué par les entreprises, les employés tentèrent également de trouver des solutions au problème du logement, à travers la création de coopératives en application de la loi sur les habitations à prix modéré de 1911 et 1921. Dans ce domaine, on relève des exemples de mise en pratique à Saragosse et à Madrid ${ }^{39}$. Ce mouvement associatif dans le secteur ferroviaire n'a néanmoins pas acquis l'importance qu'il allait atteindre par la suite, dans les années 1950, 1960 et 1970. Au cours de ces trois décennies sont apparues des coopératives ferroviaires liées à la construction de logements, dont beaucoup furent promues par les représentants locaux de l'église catholique et les Hermandades Católicas Ferroviarias (amicales catholiques ferroviaires) qui se constituèrent sous la dictature de Franco.

Après la guerre civile, époque à laquelle nombre de ces villages subirent de nombreux dégâts matériels en raison de leur proximité de la ligne de front, RENFE, de création récente, se donna pour obligation de résoudre le grave problème du logement qui concernait de nombreuses familles et, surtout, celles résidant dans les villages ferroviaires ${ }^{40}$. À cet effet, elle compta sur l'appui direct de l'État. À travers le décret du 26 avril 1944, RENFE et Explotación de Ferrocarriles por el Estado (EFE) obtinrent d'être classées dans la catégorie « entreprises de construction ». Dès lors, elles eurent la possibilité de présenter des projets à l'Instituto Nacional de la Vivienda (Institut national du logement) pour la construction de logements en location destinés à leurs agents ferroviaires, et de jouir des avantages légaux octroyés par la

37- Archivo General de la Administración (AGA), OP, 24/ 10.556, année 1926.

38- Voir Muñoz Rubio (1995), p. 32-37 et Domínguez Pedrera (1993), p. 163-195.

39- En ce sens, nous avons des preuves documentaires de l'existence à Saragosse en 1931 de la Cooperativa de Casas Baratas Unión de Funcionarios de los Ferrocarriles y Tranvías de España (AHF, C-0023-01/6. Également à Madrid en 1923, la Sociedad de Casa Baratas El Hogar Ferroviario, in Sambricio (2003), p. 132-133.

40- Memoria RENFE, année 1942, p. 248. 
loi du 19 avril 1939 sur les logements protégés, dont la possibilité d'obtenir des emprunts auprès de l'Instituto Nacional de Previsión (Institut national de prévoyance) pour financer la construction ${ }^{41}$. Les logements étaient offerts soit en régime de gratuité au personnel ayant droit à occuper un logement ${ }^{42}$, ou en régime de location aux autres employés qui pouvaient en profiter. Ces derniers payaient un loyer qui s'élevait au douzième du salaire de chaque travailleur ${ }^{43}$. Par la suite, en 1955 et avec le soutien de la nouvelle législation sur les logements protégés ${ }^{44}$, RENFE élabora un plan général de construction de logements s'élevant à 7000 unités ; un total de 2820 avait déjà été construit ou commencé sous le régime des logements protégés en $1955^{45}$. Sur le total de 7000 logements envisagés, en 19624503 étaient terminés, 648 étaient en construction, 109 étaient adjugés sur marché, 104 projets étaient rédigés et 1636 logements faisaient partie de futurs programmes ${ }^{46}$.

Comme on le soulignait dans les paragraphes précédents, la construction de logements pour le personnel ferroviaire connut un nouvel essor à partir des années 1950, lorsque furent créées dans toute l'Espagne différentes coopératives constituées par des employés qui contribuèrent pour leur part à remédier au problème du logement et à faciliter l'accès à celui-ci en régime de propriété. Il est certain qu’à l'exception de la gare Linares-Baeza, le phénomène de coopératisme

41- Instituto Nacional de la Vivienda (1947), p. 142-144. De même que RENFE, d'autres organismes officiels comme le Patronat des maisons militaires, la direction générale de la Garde civile, les mutualités des ministères de la Justice et des Finances, les associations de bienfaisance des employés des Postes et des Télécommunications, l'Institut social de la Marine, l'Institut national de colonisation, entre autres, ont commencé à jouir de ces mêmes avantages.

42- L'article 199 de la réglementation nationale du travail de la RENFE, publié au Journal officiel espagnol (BOE) du 14 janvier 1945, spécifiait les catégories professionnelles ayant droit à un logement gratuit. Il s'agissait des suivantes : chefs de gare; agents de circulation responsable des haltes, voies de remisage et quais de chargement; chefs de dépôt ou de réserve ; chef d'équipe de la voie ; ouvrier principal chargé de sous-station.

43- RENFE, Circulaire numéro 90 de la direction générale du 31 décembre 1945.

44- Décret loi du $1^{\text {er }}$ juillet 1955 pour la construction de 55000 logements à loyer limité dans un délai de 5 ans.

45- Memoria RENFE de l'année 1955, p. 59.

46- RENFE, Département du personnel et de l'assistance sociale, Rapport sur l'exercice 1962. 
ne toucha pas les villages ferroviaires puisqu'il se focalisait principalement sur les capitales de province et les villes de taille moyenne ${ }^{47}$.

Le problème du logement dans les villages ferroviaires commença à perdre de son importance avec la disparition de la vapeur au sein de RENFE, et l'exode massif de travailleurs qui s'ensuivit. À partir de ce moment, un dépeuplement progressif s'est produit. Des 48159 habitants qui résidaient dans des villages, hameaux et quartiers ferroviaires en 1960, on est passé à 28713 habitants en l'an $2000^{48}$, et il existe même des cas, comme Plasencia-Empalme (Monfragüe) ou Moreda, où le dépeuplement a été pratiquement total.

\section{L'éducation dans les villages ferroviaires}

Un autre aspect d'importance indiscutable dans le processus de consolidation des villages ferroviaires a été l'établissement de centres scolaires pour les enfants des agents qui y étaient détachés. Les principales compagnies ferroviaires privées (NORTE, MZA, MCP et ANDALUCES) ont mis en place des institutions d'enseignement pour les enfants de leurs employés.

NORTE établit ce service en 1868, et Valladolid fut le premier centre destiné à l'enseignement de culture générale pour les ouvriers, mais aussi à la formation professionnelle des enfants des agents qui accédaient à l'apprentissage. À ce premier centre s'ajoutèrent par la suite ceux de Barruelo (Palencia), Surroca (Gérone), Castejón de Ebro (Navarre), La Encina (Alicante) et Las Matas (Madrid) ${ }^{49}$, qui étaient tous des villages ferroviaires et miniers - ferroviaires.

47- Dans le «Rapport sur la situation actuelle des coopératives ferroviaires de logements » élaboré en octobre 1966 par le Département du personnel de RENFE, le nombre de coopératives ferroviaires de logements déclarés comme "protégés » par le conseil d'administration de RENFE s'élevait à 76, dont 48 étaient situées dans des capitales de province (Barcelone, Bilbao, Madrid, Saint-Sébastien, Valence, Valladolid, Albacete, Burgos, Grenade, Málaga, Pampelune, Salamanque, Santander, Séville, Huelva, Oviedo, Vitoria, León, Jaén, Alicante, Guadalajara, Orense, Tarragone, Saragosse, Cáceres, Gerona, Palencia, Teruel, Logroño et La Corogne), certaines capitales régionales disposant de plus d'une coopérative, et les 28 restantes dans des villages et des villes de taille moyenne (Alcázar de San Juan, Linares-Baeza, Puente Genil, Vigo, Aranda de Duero, Ponferrada, Reus, Ripoll, Utrera, Alsasua, Barbastro, Jerez de la Frontera, La Roda de Andalucía, Vich, Zafra, Zumárraga, Águilas, Algésiras, Miranda de Ebro, Medina del Campo, Monforte de Lemos, Tarrasa, Beasáin, Mérida, Blanes, Olaveaga, Tolosa et Badalona). Ce rapport précisait en outre qu'au mois d'octobre 1966, le nombre de logements construits et en construction par les différentes coopératives ferroviaires s'élevait à 2600 . De plus, 4600 autres logements étaient en projet.

48- Cuéllar Villar, Jiménez Vega, De Luis Roldán et Polo Muriel (2003).

49- Memorias de Norte, années 1912 et 1926. 
Dans les années 1880, la compagnie MZA avait institué ce service dans la mine de La Reunión, au sein de la municipalité alors sévillane de Villanueva del Río, et à Almorchón (Badajoz). En 1904, le centre scolaire de la mine de La Reunión offrait une instruction générale à 300 fils de mineurs et cheminots qui étaient établis dans ce centre de population ${ }^{50}$. À l'instar de ce qui s'est produit avec NORTE, d'autres centres éducatifs se sont également ouverts dans des agglomérations ferroviaires (La Encina, Chinchilla, Baeza et Espeluy) et dans des localités ou apparaissait une forte présence du groupe professionnel, comme ce fut le cas à Aranda de Duero (Burgos).

Les autres compagnies ouvrirent également des écoles dans les localités qui relevaient d'elles. La compagnie du MCP commença la construction de chapelles-écoles dans les localités de Plasencia-Empalme et Arroyo-Malpartida dans les années 1908 et 1909 respectivement, qui devaient ouvrir leurs portes en $1910^{51}$. Les premiers projets d'écoles dépendant de la compagnie du ANDALUCES datent de l'année 1915, époque à laquelle ont été définies les bases de la gestion conjointe, entre MZA et ANDALUCES, d'une école dans la localité d'Espeluy ${ }^{52}$. Par la suite, entre 1925 et 1930, elle devait ouvrir son propre centre scolaire à Moreda (Grenade) et en projeter un autre à Bobadilla (Málaga) ${ }^{53}$.

Au cours de la période de gestion des anciennes compagnies, l'éducation était généralement à la charge de maîtres d'école ou du prêtre de l'église ou de la chapelle de la localité et, dans les centres miniers ferroviaires de Barruelo et de La Reunión, cette fonction fut confiée au cours des années 1920 à l'ordre des frères maristes ${ }^{54}$.

La guerre civile touchait à sa fin et, une fois la RENFE constituée, la nouvelle entreprise nationale se donna pour objectif d'unifier et de réglementer le service éducatif qui était autrefois réalisé par les anciennes compagnies. Ainsi, en 1943, elle confia au Service d'assistance sociale la gestion de toutes les écoles créées par les anciennes compagnies. La première tâche de ce service fut la rédaction d'un règlement qui fut approuvé cette même année par la direction générale de l'enseignement élémentaire ${ }^{55}$. Parmi les quatorze écoles directement soutenues par

50- Memoria de MZA, année 1904, p. 16.

51- Memoria de MCP, années 1908 (p. 12), 1909 (p. 13) et 1910 (p. 18).

52- AHF, C-0199-01.

53- Memorias de Andaluces, années 1923 à 1929.

54- AHF, S-0172-032b.

55- Des bibliothèques ambulantes ont également été établies dans les villages d'Algodor, Baeza, Los Rosales, Chinchilla et Almorchón. RENFE, Memoria de Explotación del año 1943, p. 265-268. 
RENFE en 1943, treize étaient situées dans des villages ferroviaires. Ces centres scolaires accueillaient 1487 élèves (733 garçons et 754 filles) et comptaient sur un corps de trente maittres d'écoles (douze hommes et dix-huit femmes) dont le salaire était à la charge de RENFE et qui bénéficiaient d'un logement gratuit au sein des localités où ils étaient affecté $^{56}$. Dix ans plus tard, en 1953, le nombre d'écoles de RENFE s'élevait déjà à 19 et l'on y éduquait un total de 1684 élèves (848 garçons et 837 filles $)^{57}$. Dès cette époque, on détecte une diminution progressive du nombre d'élèves, principalement due à la réforme de l'éducation lancée en Espagne dans les années $1970^{58}$ ainsi qu’à la diminution déjà citée de la population recensée dans ces foyers de population. Cette diminution des élèves justifiera, à la longue, la fermeture de la plupart des centres scolaires ${ }^{59}$. Quoi qu'il en soit, aujourd'hui sont toujours ouverts les centres scolaires du quartier ferroviaire de Guadix et celui du village ferroviaire de La Encina, tous deux dépendant désormais de leurs municipalités respectives.

Le niveau académique qu'offraient les écoles, aussi bien du temps des anciennes compagnies que par la suite, n'atteignait que le stade élémentaire. Lorsque les élèves atteignaient l'âge réglementaire de 14 ans, ils devaient abandonner le centre scolaire. L'enfant qui souhaitait poursuivre ses études était donc obligé de quitter la localité.

« ...lorsque nous parvenions à un âge qui permettait d'entrer à la RENFE, l'objectif était de suivre la trajectoire du père, mais pour ce qui est d'étudier... nous autres, nous étions quatre frères en plus de nos parents, et si l'on gagnait 5,50 ou 6 pesetas, à cette époque il n'y a avait pas de possibilité d'étudier. Qui étudiait ? Les gens qui avaient de l'argent. Et ceux qui étaient dans une capitale, ce n'est pas qu'ils étudiaient jusqu'au baccalauréat, mais ils allaient à d'autres écoles particulières, qui permettaient de dépasser un peu ce qu'ils avaient appris. Il y en avait très peu. Mais de mon époque, moi je ne connais personne qui ait fait un cursus universitaire ${ }^{60} . »$

56- RENFE, Memoria de Explotación del año 1943, p. 266.

57- RENFE, Memoria de Explotación del año 1953, p. 47.

58- Capitán Díaz (2002).

59- Domínguez Pedrera (1993) ; Jiménez Vega et Polo Muriel (1999).

60- Témoignage de J.L.T.S. (77 ans) du village ferroviaire d'Espeluy (Jaén). 
Il y eut des cas où la proximité entre le village et la capitale de province ou une ville de taille moyenne permettait aux enfants des cheminots un aller-retour dans la journée. À cet effet, RENFE est même allé jusqu'à mettre en circulation des trains spéciaux.

«Mon fils allait et venait d'Arroyo à Cáceres tous les jours. On mettait à leur disposition un train le matin à sept heures pour les emmener à l'école à Cáceres. Le train, qu'ils appelaient "le train des enfants", revenait ensuite en milieu de journée, à une heure et quelques, et c'est comme ça que descendaient tous ceux qui venaient du collège ou de l'école... ${ }^{61}$.»

C'est aussi à partir de l'âge de quatorze ans que les fils des cheminots qui restaient dans la localité, soit parce qu'ils ne voulaient pas poursuivre leurs études, soit parce que les ressources économiques des parents ne leur permettaient pas de payer un hébergement dans la ville la plus proche ou à la capitale, commençaient à apprendre le métier de leurs pères en vue d'une future incorporation au sein de l'entreprise. Nous considérons cette tendance à connaittre le métier du père comme une spécificité de l'ambiance purement ferroviaire que l'on respirait dans la localité et de la proximité entre les centres de travail et ceux de résidence. Cet enseignement professionnel «non réglementé » contribua à la reproduction professionnelle de l'emploi du père, à la transmission d'une certaine culture du travail et à l'apprentissage d'un métier qui, bien que ne présentant pas les carctères de l'artisanat, pouvait être transmis par le père ou les voisins aux enfants. L'enseignement réglementé n'offrait pas ces connaissances, mais il existe cependant le cas de personnes qui se souviennent, à l'école du village, d'avoir appris leurs premières notions en matière ferroviaire.

" ...à l'école, on nous apprenait la grammaire, l'arithmétique, la géométrie, l'histoire et les sciences, mais nous avions aussi d'autres matières où l'on apprenait, par exemple, gare après gare, toutes les lignes ferroviaires. Lorsque je suis entré à RENFE, je connaissais toutes les lignes ferroviaires d'Espagne, aussi bien locales que les correspondances. Je ne sais pas comment notre maitre d'école, qui s'appelait Don Alfredo, nous a inculqué cela. Je ne sais pas si c'était pour que cela soit ensuite plus facile pour nous..., parce que bien entendu si l'on voulait être cheminot dans la catégorie de facteur, il fallait s'inscrire dans une académie particulière à Málaga ou à Madrid et passer les examens par correspondance, et j'y ai été moi aussi pendant longtemps ${ }^{62}$. »

61- Témoignage de N.V.P. (84 ans) du village ferroviaire d'Arroyo-Malpartida (Cáceres).

62- Témoignage de J.L.T.S. (77 ans) du village ferroviaire d’Espeluy (Jaén). 
En ce qui concerne l'influence exercée dans les villages ferroviaires par les centres de travail pour l'apprentissage du métier ferroviaire, un certain nombre de témoignages exprime des opinions différentes.

« ...les centres de travail [...] étaient exclusivement réservés au personnel qui travaillait. Il était pratiquement inadmissible qu'un garçon puisse arriver à [aller] voir son père au travail. Quel que soit le domaine de travail, qu'il s'agisse des ateliers ou des bureaux, [...] les enfants et l'épouse des agents devaient toujours respecter la zone de travail, et nous ne nous approchions donc pas de ces endroits ${ }^{63} . »$

En revanche, un autre témoin affirme:

«...je sortais de l'école et $j$ 'allais voir mon père ; mon père travaillait sur le parcours [el recorrido, services d'entretien], il était Visiteur principal, ...alors je me rendais sur le parcours - le parcours, c'était le service des vapeurs et les voitures et tout ça, c'est-à-dire le matériel remorqué - et là je voyais comment il faisait les choses, et [mon père] me disait: Observe !! Observe mon fils !, au cas où demain tu devrais, toi aussi, faire ce métier. Et tous les garçons allaient voir leurs pères et certains apprenaient, et y passaient tout leur temps... ${ }^{64}$.»

Une autre façon d'avoir accès à ces connaissances était la préparation qu'un employé d'exception, avantagé ou altruiste, offrait aux enfants des employés autrefois, comme le souligne le témoignage suivant:

«...Toute ma préparation... ferroviaire, je l'ai faite à travers les agents qui travaillaient ici dans la catégorie de facteur, qui voulaient nous apporter et apprendre quelque chose qu'ils savaient et qu'ils apportaient indubitablement [...], j'ai donc reçu une préparation à travers un facteur qu'il y avait dans cette gare ; ça n'est pas qu'il se consacrait à çà, mais c'était pour lui un grand orgueil de préparer précisément les fils des cheminots. Et je ne suis pas le seul à avoir été préparé comme ça : c'est aussi le cas d'autres garçons, aussi des fils d'agents de cette entreprise, qui cherchaient précisément à s'ouvrir une voie professionnelle à travers l'incorporation au sein de la RENFE. C'était un grand homme, un grand altruiste, qui s'occupait beaucoup des enfants des employés [...] Ce qu'il faisait, c'est qu'il prenait un petit groupe de six à huit garçons qui préparaient précisément ce type de concours, et dans un petit local qui n'était pas de la RENFE, qu'on lui céda, il donnait des cours. Il ne percevait absolument rien pour communiquer ses connaissances ; la seule chose que nous faisions, c'était de lui payer le montant de la location de ce local ${ }^{65}$.»

63- Témoignage de V.M.R. (78 ans) du village ferroviaire de Plasencia-Empalme (Cáceres).

64- Témoignage de C.T.F. (85 ans) du village ferroviaire d'Arroyo-Malpartida (Cáceres).

65- Témoignage de V.M.R. (78 ans) du village ferroviaire de Plasencia-Empalme (Cáceres). 
Les caractéristiques en matière d'éducation dans les villages ferroviaires qui ont été soulignées dans ce chapitre ne sont qu'un premier point de réflexion et d'étude, qui devra être approfondi pour connaitre le véritable rôle de pépinière que ces sites d'exploitation ferroviaire ont joué pour des générations successives de cheminots.

\section{La formation religieuse}

Comme nous le disions dans le chapitre précédent, l'instruction qui était donnée à l'époque des anciennes compagnies était pratiquement entièrement liée à l'Église catholique, qui se chargeait de l'église ou de la chapelle existant dans la localité. Les prêtres inculquaient les connaissances correspondant à l'enseignement élémentaire, en endoctrinant les enfants de ces villages ferroviaires. De même que l'existence d'une école permit la consolidation de foyers de population nettement ferroviaires, l'existence d'une église ou d'une chapelle aida également à ce que ce processus se perpétue au cours du temps.

La plupart des églises qui se sont construites dans les villages ferroviaires ont été érigées par les entreprises elles-mêmes, qui se voyaient également dans l'obligation de faciliter à leurs employés le respect de leurs devoirs religieux ${ }^{66}$. On est même parvenu à construire des églises avec les donations aux diocèses ou aux compagnies elles-mêmes de propriétaires terriens locaux. Parmi les exemples répondant à ce cas de figure, citons la Gare de Linares-Baeza et Espeluy (Jaén), Guadix (Grenade) ou Port-Bou (Gérone), où l'entrepreneur du Réseau catalan du MZA, Claudio Planas, finança la construction d'un temple néogothique sur les emprises de l'exploitation ferroviaire.

À partir de la création de RENFE a débuté la reconstruction de nombreuses églises qui avaient été utilisées à d'autres fins pendant la guerre civile. Le service religieux a été rétabli dans toutes les localités, et un processus de catéchisation a été engagé sur tout le réseau ferroviaire, qui concernait tous les niveaux professionnels existant au sein de l'entreprise ${ }^{67}$. Pour mettre en œuvre toute la force évangélisatrice du nouveau régime dans l'entreprise, il fut créé, au sein de la Division du personnel et de l'assistance sociale, un Secteur du culte et d'assistance spirituelle. Cet appendice pastoral de l'entreprise fut chargé de prêter assistance spirituelle au personnel et de lui faciliter l'exercice de ses devoirs religieux. Ce Secteur du culte fut chargé d'organiser des séries d'exercices

66- L'article 154 du Règlement pour le service des gares de la Compañía de los Ferrocarriles de Tarragona a Barcelona y Francia de 1887 stipulait que l'entreprise devait accorder à l'employé le temps nécessaire pour assister aux services religieux.

67- Memoria de RENFE, année 1944, p. 417. 
spirituels, d'organiser des missions évangélisatrices et des conférences dans les propres emprises de l'entreprise, et d'établir le culte religieux dans les gares ${ }^{68}$. Les services de culte et d'éducation allaient prendre en charge une grande partie de la vie sociale des villages ferroviaires pendant tout le franquisme, puisqu'ils étaient responsables de l'organisation des premières communions et autres festivités religieuses telles que la fête des Rois mages (avec leurs distributions de jouets de la part de l'entreprise), la Fiesta de las Espigas et Fiesta del Árbo ${ }^{69}$. Toutes ces festivités s'appuyaient sur des apports financiers directs de l'entreprise publique. À ces services dépendant de l'entreprise s'ajouteraient plusieurs années plus tard les Agrupaciones de la Adoración Nocturna $a^{70}$ et les Hermandades Católicas Ferroviarias qui ont été créés sur tout le réseau ferroviaire, des confréries qui sont toutes deux le fruit du travail apostolique promu directement par l'entreprise, avec le soutien exprès de l'Église catholique.

De même que les aspects éducatifs, tout ce qui concerne les aspects religieux dans la vie d'une localité mérite d'être étudié très en détail pour connaître le rôle joué, non seulement par l'Église catholique mais aussi par les associations et confréries spirituelles qui sont apparues, pendant l'après-guerre et au cours des décennies suivantes, jusqu'à l'avènement de la démocratie en Espagne.

\section{Autres services}

Au début de ce chapitre, nous avons souligné l'importance accordée par les services médicaux de toutes les entreprises aux conditions de vie dans les logements et les autres bâtiments des localités, en raison des conséquences que celles-ci pourraient avoir sur la propagation de maladies contagieuses. Cela conduit les services médicaux des entreprises à demander à leurs directions des améliorations continues au sein des installations ${ }^{71}$.

68- Memoria de RENFE, année 1943. Division du personnel et de l'assistance sociale. Secteur du culte et de l'assistance spirituelle.

69- Les revues spécialisées Ferroviarios et Via libre contiennent un grand nombre de références sur la tenue de ces événements et festivités.

70- Le premier regroupement d'Adoración Nocturna connu en Espagne après la guerre civile a été créé dans le village ferroviaire d'Espeluy (Jaén). Memoria de RENFE, 1943, p. 265.

71- Un bon exemple serait un dossier de l'année 1904 relatif à l'agrandissement du dortoir des conducteurs de la gare d'Almorchón, qui précise les conditions que celui-ci offrait au personnel. Cet espace comportait 16 lits distribués avec une faible séparation entre eux, et ne réunissait pas les conditions d'hygiène élémentaires. Ce dossier conserve une lettre du médecin principal de la compagnie ferroviaire proposant des mesures assurant la ventilation et la salubrité de l'espace, qui furent approuvées par Süss, alors directeur de MZA. AHF, C-0930-01/14. 
La maladie infectieuse la plus fréquente dans ces localités fut le paludisme. Les foyers paludiques affectèrent avec une certaine intensité la population ferroviaire résidente depuis le début de l'exploitation des villages d'Almorchón, Algodor, Vadollano ${ }^{72}$, Villanueva del Río y Minas (La Reunión) et Plasencia-Empalme, entre autres. L'action préventive développée par les anciennes compagnies, et par la suite celle entreprise par RENFE, a permis l'élimination progressive de ces foyers infectieux. Dans un rapport de 1936 sur l'effet du paludisme sur le réseau de MZA, il était affirmé que la maladie n'affectait plus que 1,5\% de l'effectif et que, par rapport à 1926, le nombre des agents infectés avait été réduit de moitié. La compagnie soulignait que cette réduction avait été obtenue sur la base de la mise en application d'une politique préventive qui consistait en l'administration d'un traitement adéquat (sulfate de quinine en comprimés), l'ouverture de nouveaux dispensaires médicaux dans les lieux de plus grande activité de la maladie, la plantation d'eucalyptus aux environs des gares et l'installation de mailles métalliques aux fenêtres des bâtiments et logements ferroviaires ${ }^{73}$. Cependant, la guerre civile vint interrompre cette tendance à la baisse et provoqua, pour des raisons évidentes, une recrudescence de la maladie ${ }^{74}$. De fait, à Plasencia-Empalme, il fut impossible de maîtriser l'épidémie jusqu'au début des années $1950^{75}$.

Mis à part ce grand fléau qui altéra longtemps la vie quotidienne au sein des villages ferroviaires, les services médicaux les dotèrent pratiquement tous de cabinets médicaux et centres de soins destinés à garantir la santé de tous les résidents. Dans les lieux où la population était plus

72- Le rapport élaboré par l'ingénieur en chef des Mines de Linares en juin 1886 pour la Commission des réformes sociales témoigne de réflexions fort éclairantes à cet égard.

73- Compañía de los ferrocarriles de Madrid, Zaragoza y Alicante (1936).

74- Une lettre remise le 13 mai 1940 par le médecin en chef de la section au chef du Service du personnel et de l'assistance sociale l'informe sur l'épidémie de paludisme et de gale qui avait été observée au sein de la population ferroviaire d'Almorchón. Le nombre d'infectés s'élevait à 300, tous appartenant aux 100 familles résidantes. Le médecin chef proposait que le wagon sanitaire revienne la semaine suivante pour apporter les médicaments suffisants en vue de traiter la population affectée (pommade Helmerich et quinine). Il discutait en outre les causes de l'apparition du paludisme : croupissement des eaux provenant de l'évacuation des égouts et par les cratères générés par les bombardements subis par le village pendant la guerre civile. Et il demandait au Service voie et travaux d'assécher les eaux marécageuses dans les plus brefs délais.

75- Memoria RENFE, année 1951, p. 99. 
grande et avec un plus haut risque d'accidents, comme ce fut le cas de Villanueva del Río y Minas, MZA construisit un hôpital qui vint pallier en partie les fréquents sinistres qui se produisaient dans les différents puits en exploitation. Dans les cas où il n'existait pas de cabinet médical la couverture médicale était garantie par le parcours régulier que réalisaient les trains sanitaires sur leurs réseaux ferroviaires respectifs.

Le train sanitaire n'était pas le seul qui arrivait régulièrement sur les faisceaux de voies des villages et offrait des services d'assistance aux travailleurs et à leurs familles. Parmi les autres éléments qui animaient le passage des jours au sein des localités, citons le train de paye et le train économat.

Toutes les compagnies ferroviaires instituèrent un service d'économat sur tous leurs réseaux. Au sein de NORTE il fonctionna dès 1879 et, jusqu'en 1919, il fit partie du Service des magasins généraux de l'entreprise. À compter de cette date et jusqu'en mars 1933, il fonctionna comme une coopérative, puis ensuite à nouveau comme un économat. Dans notre domaine d'étude, il y eut des succursales d'économats à Venta de Baños, à Barruelo et à Surroca ${ }^{76}$.

L'économat de MZA commença à fonctionner en 1903. En 1919, il fut distingué comme un service indépendant, pour revenir à la situation première en 1932. C'est en 1936 qu'a été inaugurée la succursale d'Almorchón, qui fut ensuite détruite pendant la guerre ${ }^{77}$.

L'économat de MCP fut inauguré le premier mai 1917. Il fut créé selon une formule d'association, dont le capital était composé de l'apport de 1858 agents à raison de 25 pesetas chacun. C'est ainsi que fut créée l'A Asociación Cooperativa de los Agentes de la Compañia de Explotación de los Ferrocarriles de Madrid a Cáceres y Portugal y del Oeste de España dont la mission était de fournir à ses membres de Madrid et de la ligne de Cáceres des produits comestibles et du combustible. En 1928, après la fusion des autres lignes et la création de la Compañía Nacional de los Ferrocarriles del Oeste de España (OESTE), le titre fut changé en Sociedad en Cooperativa de los Ferroviarios del Oeste de España ${ }^{78}$. Finalement, l'économat de ANDALUCES, situé à Málaga, fonctionna depuis sa création en 1901 en tant qu'économat.

76- RENFE (1942), Economato, p. 2.

77- Ibid., p. 16-17.

78- Ibid., p. 33-34. 
Après la création de RENFE, la nouvelle entreprise publique unifia en un seul économat tous les services homonymes qui avaient fonctionné dans les anciennes compagnies ${ }^{79}$. Néanmoins, dans toutes les agglomérations, il existait de petits magasins et épiceries, et des licences ont été octroyées pour ouvrir des tavernes et autres types de commerces. Cela résolvait le problème de l'approvisionnement quotidien en produits de première nécessité que l'économat ne permettait pas de résoudre en raison de la périodicité mensuelle avec laquelle il effectuait les approvisionnements aux employés. L'existence d'une coopérative de consommation a également été attestée à Almorchón ; malgré sa courte vie, elle parvint à adopter la dénomination de Sociedad Cooperativa Benéfica Ferroviaria de Almorchón ${ }^{80}$.

\section{L'organisation administrative, les forces de l'ordre et la vie sociale}

Tous les services ici décrits garantissaient les besoins élémentaires de la population ainsi que d'autres aspirations de nature spirituelle ou festive, bien que toujours liées à des activités religieuses. Mais les moments de loisir, dans un village ferroviaire, étaient organisés et animés par les employés eux-mêmes et par leurs familles. Dans ce domaine intervenaient aussi généralement les commerçants et les concessionnaires des auberges, qui organisaient dans leurs commerces des cabarets improvisés (s'il s'agissait de petits magasins), des bals, des salles de projection de films et tous types d'activités destinées à animer les loisirs de la population résidente. Les membres du personnel ferroviaire eux-mêmes parvinrent à s'organiser en sociétés de loisirs, à travers lesquelles ils créèrent des clubs et des petits casinos, qui étaient surtout pris en charge par les cotisations que versaient leurs membres.

La gare en elle-même et le va-et-vient continu des trains et des voyageurs constituaient déjà en soi l'un des plus grands attraits de la vie quotidienne du village. L'environnement du bâtiment voyageurs et les quais de la gare devenaient pour tous les habitants un lieu de promenade obligé du dimanche. Et pas seulement pour eux, mais encore pour les habitants des localités voisines. Cette attraction s'amplifiait plus encore au moment des fêtes et foires locales, quand la gare était le lieu d'arrivée des forains, négociants de bétail, etc.

79- L'étude sur l'implantation de ce service se trouve dans l'ouvrage RENFE (1942, Economato.

80- AHF, C-0530-06. 
Les fêtes qui se tenaient et sont toujours organisées dans certains des villages ferroviaires avaient habituellement un motif religieux : el Cristo de la Buena Muerte à Algodor, la Virgen de Belén à Almorchón, San Luis Gonz̧aga à Chinchilla, San José Obrero à Maçanet-Massanas et Las Matas, pour ne citer que les plus représentatives. D'autres sont aujourd'hui devenues des festivités à motivation plus profane, comme c'est le cas d'Espeluy avec la fête du Jour de l'émigré. L'organisation de toutes ces fêtes comptait toujours sur la participation de l'entreprise, sous une forme ou sous une autre, soit par l'apport d'argent au groupe qui l'organisait ou à la municipalité dont dépendait le village, soit par la cession de matériel ou d'heures de travail pour que certains des employés des différents services préparent les chars, construisent les arcs triomphaux ou montent les arènes avec des traverses pour les combats de vachettes.

Étant donné que la plupart des services essentiels étaient à la charge des entreprises, la célébration des fêtes était l'occasion de se faire voir pour la personne qui représentait la municipalité dont dépendait le village. La plupart des villages ferroviaires dépendaient administrativement de la municipalité au sein de laquelle ils étaient insérés. Il existe peu d'exemples de séparation administrative ; Castejón (Navarre) est sans doute le seul à avoir obtenu un tel statut en se séparant de Corella en 1928. D'autres l'on tenté par la voie des urnes après l'avènement de la démocratie, mais n'y sont pas parvenus : tel est le cas de Las Matas, dépendant de la municipalité de Las Rozas (Madrid) ${ }^{81}$; il y en a même qui ont à leur actif de longues décennies à la recherche de la séparation de leur municipalité de tutelle, comme c'est le cas de Puerto de Sagunto ${ }^{82}$. En revanche, il existe des cas où certains, en raison de leur emplacement et du dynamisme économique généré par le transport ferroviaire, ont réussi à détrôner les foyers de population historiques dont ils dépendaient. Les exemples les plus représentatifs sont ceux de Venta de Baños (Palencia), vis-à-vis de Baños de Cerrato, Villanueva del Río y Minas (Sevilla), vis-à-vis de Villanueva del Río, et CanfrancGare, vis-à-vis de Canfranc ${ }^{83}$ ainsi que Puerto de Sagunto - déjà mentionné - vis-à-vis de Sagunto. En tout état de cause, dans la plupart des cas ces sites ont habituellement atteint le statut d'entités locales de moindre importance, comme il arrive à Linares-Baeza (Jaén), ArroyoMalpartida (Cáceres) et La Encina (Alicante).

81- Jiménez Vega et Polo Muriel (2003).

82- Llueca Úbeda (1996).

83- Dans ce dernier cas, l'incendie qui a désolé le village historique de Canfranc au début des années 1940 y a également contribué. 
Quant au poste de maire ou de représentant municipal, nous avons constaté dans les entretiens qu'il était généralement occupé par un employé (pas nécessairement celui de plus haut rang), le commerçant de la localité ou le concessionnaire de l'auberge de la gare, comme ce fut le cas de Chinchilla, où il se trouve qu'il s'agissait également du médecin de la localité.

Finalement, exception faite des agglomérations qui parvinrent à se constituer en communes ou en entités locales de moindre importance, où la police municipale garantissait l'ordre public sur les lieux, dans la période RENFE, il s'est créé, au sein de la Garde civile, un groupe d'enquête et de surveillance qui fonctionna au sein de l'entreprise publique jusqu'à la fin de la dictature de Franco.

Ce corps spécial, que l'on dénommait communément la brigadilla au sein de RENFE, était composé d'une direction de service à deux branches : la première (Enquêtes) se consacrait à tout ce qui concernait la surveillance des carrefours ferroviaires de plus grand intérêt (parmi lesquels se trouvaient tous les villages ferroviaires), comme les centres de formation de trains et de dégroupage de marchandises, gares de triage, centre et transferts pour éviter les vols de marchandises et la perpétration d'actes subversifs ou de sabotage. La seconde (Surveillance) avait pour mission de « connaître l'atmosphère régnant au sein du personnel ferroviaire en vue d'évaluer les opinions et ainsi surveiller les manœuvres obscures et subversives » (sic). Les membres de ce groupe de surveillance avaient l'obligation de communiquer toute information d'intérêt relative à des problèmes professionnels que tentaient « d'exploiter des agents inspirés par des puissances étrangères » (sic $)^{84}$.

\section{Conclusions}

Aujourd'hui, les villages ferroviaires ne sont plus que le reflet de ce qu'ils furent il n'y a pas encore si longtemps. Comme le montre ce travail, à leur époque de plus grande splendeur, ils avaient une notable importance économique et sociale. D’une part, leur phase d'expansion correspondit au moment où le chemin de fer avait encore besoin d'une main-d'œuvre nombreuse pour son exploitation. Ceci leur permit d'accroître progressivement leur population et de générer dans leur entourage un intéressant développement économique dont ont bénéficié non seulement les cheminots, mais aussi d'autres activités telles que le

84- Guardia Civil, Memorias del Grupo de Investigación y Vigilancia en la RENFE de la Guardia Civil años 1962 y 1963, p. 2-3. 
commerce, les services, la construction et les loisirs, donnant ainsi lieu à une phase de développement industriel sans précédent. Ils constituèrent d'autre part un cadre particulièrement important de modernisation sociale. Jusqu'à la récente explosion des médias, le chemin de fer était le meilleur diffuseur de nouvelles et d'informations. En ce sens, ces villages ferroviaires assimilèrent rapidement les améliorations qui leur parvenaient à travers la dotation des « logements sociaux » et la prestation de services éducatifs et médicaux permanents. Tout ceci dans un contexte proche du mode de vie rural, où il n'était pas habituel de trouver des améliorations et des progrès proches d'une vie urbaine. Le village ferroviaire devenait ainsi un univers intégré et suffisamment équipé pour ne pas dépendre excessivement de l'extérieur.

L'abandon et la détérioration qu'ils connaissent aujourd'hui ne font que souligner l'importance de leur vie antérieure. Entre les murs de leur parc immobilier et près des voies de chemin de fer pratiquement désertes de ces foyers de population, résonnent encore les voix et les rythmes d'une activité autrefois frénétique et aujourd'hui définitivement oubliée.

\section{Bibliographie}

\section{Sources primaires}

Archivo Histórico de Andaluces, déposé à la Fundación de los Ferrocarriles Españoles.

Archivo Histórico de M.Z.A, Secciones de Infraestructura y de Secretaría General del Consejo, déposé à la Fundación de los Ferrocarriles Españoles.

Caminos de Hierro del Norte de España (1915-1941) : Memorias de explotación.

Caminos de Hierro del Norte de España (1913) : Esquemas de estaciones de la línea Madrid-Irún.

Caminos de Hierro del Norte de España (1912) : Instituciones establecidas por la Compañia de los Caminos de Hierro del Norte de España a favor de su personal y mejoras que ha concedido al mismo en los últimos años, Madrid, Imprenta Central de los Ferrocarriles.

Caminos de Hierro del Norte de España (1900): Instrucción general número 2 sobre el personal de los servicios de la compañia, Madrid.

Compañía de los Ferrocarriles de Madrid, Zaragoza y Alicante (1936) : Elpaludismo en la red de la Compañia. 
Compañía de los Ferrocarriles de Madrid, Zaragoza y Alicante (1934) : Situación del personal en la Compañia MZA (1913-1933), Madrid, Sucesores de Rivadeneyra (S.A.).

Compañía de los Ferrocarriles de Madrid, Zaragoza y Alicante (1921) : Croquis de estaciones de las lineas de Madrid a Ciudad Real y de Castillejo a Toledo.

Compañía de los Ferrocarriles de Tarragona a Barcelona y Francia (1887) : Reglamento para el servicio de las estaciones.

Compañía Nacional de los Ferrocarriles del Oeste de España (1930) : Estatuto del personal de la Compañáa, Madrid, Sucesores de Rivadeneyra (S.A.).

Ferrocarril del Tajuña (1992) : Convenio colectivo. Sector ferroviario de comisiones obreras, Madrid.

Guardia Civil, Memorias del Grupo de Investigación y Vigilancia en la RENFE de la Guardia Civil años 1962 y 1963.

Instituto Nacional de Estadística, Nomenclátores de censos de población.

RENFE, Memoria de explotación, 1941-2001, Madrid.

RENFE, Departamento de personal y asistencia social (1962) : Rapport sur l'exercice 1962.

RENFE. División de personal y asistencia social. Sector de culto y asistencia espiritual (1943) : Rapport sur l'exercice 1943.

RENFE (1942): Economato.

RENFE, Esquemas de estaciones de las líneas de Madrid a Ciudad Real, Castillejo a Toledo, Almorchón a Belmezy Aljucén a Cáceres.

\section{Études}

Miguel Artola Gallego (sous la dir. de) (1978) : Los ferrocarriles en España (1844-1943), Madrid, Banco de España, 2 vol.

Ayuntamiento de Mieres (1999): El poblado minero de Bustiello, Mieres, Ayuntamiento de Mieres.

Esmeralda Ballesteros Doncel et Tomás Martínez Vara, (2001) : «La evolución del empleo en el sector ferroviario español, 1893-1935 », Revista de Historia Económica, $19^{\mathrm{e}}$ année, automne-hiver, nº 3, p. 637-677.

Jorge Bogaerts (2000) : El mundo social de Ensidesa. Estado y paternalismo industrial (1950-1973), Avilés, Editorial Azucel.

María Paz Cabello Rodríguez (1983) : Barruelo de Santullán. La crisis de un núcleo minero, Valladolid, Universidad de Valladolid.

Albert Carreras et Xavier Tafunell (1993) : «La gran empresa en España (1917-1974). Una primera aproximación ", Revista de Historia Industrial, $\mathrm{n}^{\circ} 3$, p. $127-175$. 
Alfonso Capitán Díaz (2002) : Breve historia de la educación en España, Madrid, Alianza Editorial.

María José Casaus Ballester et José Luis Martí González (2001) : Compañia Minera de Sierra Menera : el futuro de un pasado, Teruel, Ayuntamiento de Puebla de Valverde.

Santiago Castillo (1983) : «El asociacionismo ferroviario y su génesis », Estudios de Historia Social, nos 26-27 (juil.-déc. 1983), p. 207-255.

Castro Iglesias (1999) : Ferroviarios, Madrid, Imp. Campillo Nevado, S.A. Arón Coen (2002) : Minas y mineros de Granada (siglos XIX y XX), Colección Los Libros de la Estrella, Diputación de Granada.

Francisco Comín Comín ; Pablo Martín Aceña ; Miguel Muñoz Rubio et Javier Vidal Olivares (1998) : 150 años de historia de los ferrocarriles españoles, Madrid, Fundación de los Ferrocarriles Españoles, Anaya, 2 vol.

Jordi Clua I Mercadal (2001) : Les colònies industrials, San Cugat del Vallés, Amelia Romero Editora.

Domingo Cuéllar Villar ; Miguel Jiménez Vega ; Elena De Luis Roldán et Francisco Polo Muriel (2003) : «Los poblados ferroviarios en España : historia y patrimonio », Patrimonio Cultural y Derecho, n ${ }^{\circ}$ 7, p. 159-207.

María del Carmen Domínguez Pedrera (1993) : «La estación de ArroyoMalpartida como depósito de puestos fijos ", Norba, Revista de Historia, no 13, p. 163-195.

Gracia Dorel Ferre (2003) : «Les colònies industrials catalanes dins la historia de l'habitatge social », in Museu de la Ciencia i de la Técnica de Catalunya, Actas del Congreso sobre L'babitatge obrer a Catalunya.

Diane K. Drummond (1990) : Crewe: Railway Town, Company and People, 1840-1914, Scolar Press.

Francisco Esteve et José Luis Esparcia (1991) : Historia de La Encina y su estación, Madrid, Mesidor Ediciones.

Pilar Folguera (sous la dir. de) ; Pilar Díaz Sánchez ; Pilar Domínguez Prats et José María Gago González (2003) : El mundo del trabajo en RENFE. Historia oral de la Infraestructura, Madrid, UN de Mantenimiento de Infraestructura en RENFE et Fundación de los Ferrocarriles Españoles.

Dionisia Gómez Amelia (1978) : Aldea Moret : de poblado minero a suburbio cacereño, Cáceres, Caja de Ahorros y Monte de Piedad de Cáceres.

Esmeralda González Urruela (2001) : De los tajos a los embarcaderos, Barcelona, Ariel.

Instituto Nacional de la Vivienda (1947) : Viviendas Protegidas, Madrid. Miguel Jiménez Vega (1992) : «En torno al puerto de Torralba », Vía Libre, mai 1992, p. 71-72. 
Miguel Jiménez Vega et Francisco Polo Muriel (1999) : «Aproximación al estudio de los poblados ferroviarios : el caso de Algodor », in Muñoz Rubio, Sanz Fernandez et Vidal Olivares (sous la dir. de), Siglo y medio de ferrocarril en España, 1848-1998 : Economía, Industria y Sociedad, p. 933-955.

- (2003a) : «Al encuentro del pasado de tres poblados ferroviarios en la Comunidad de Madrid : los casos de Algodor, Las Matas y Vicálvaro ", in Benegas Capote, Matilla Quiza et Polo Muriel (sous la dir. de), Ferrocarril y Madrid, historia de un progreso, p. 243-275.

Emerenciana Paz Juez Gonzalo (1992) : El mundo social de los ferrocarriles españoles de 1857 a 1917, thèse de doctorat, Madrid, Universidad Complutense.

- (2000) : Los Ferroviarios de las antiguas compañias (una historia desconocida), Gijón, Asturias, Ediciones Trea.

Ángel Larrea ; Fernando Palacios et Javier Velaza (2000) : Castejón : Álbum de fotos, Castejón, Ayuntamiento de Castejón.

Emilio Llueca Úbeda (1996) : Los intentos segregacionistas del Puerto de Sagunto, 1926-1996, Valencia, Ediciones y Promociones LAV.

Miguel Muñoz Rubio (1995): RENFE (1941-1991). Medio siglo de ferrocarril público, Madrid, Ediciones Luna.

Miguel Muñoz Rubio et Javier Vidal Olivares (2001) : «Los ferrocarriles en la historiografía española », TST, n 1, p. 81-111.

Jesús María Palomares Ibáñez (1992) : El asociacionismo minero en el primer tercio del siglo XX. El sindicalismo minero de Barruelo (1900-1936), Palencia, Diputación provincial de Palencia.

Santiago Parra De Más (1988) : Elferrocarril de Canfrancy los transpirenaicos, Madrid, Aldaba.

Pere Pascual I Domènech (1999): Los caminos de la era industrial. La construcción y financiación de la red catalana de ferrocarriles (1843-1898), Barcelona, Fundación de los Ferrocarriles Españoles/Universidad de Barcelona.

Miguel Reche Sánchez (1988) : La minería de Serón, 1870-1970. Nacimiento, desarrollo y muerte de Las Menas, Almería, Instituto de Estudios Almerienses et Club "Amigos de Serón".

Carlos Sambricio (sous la dir. de) (2003) : Un siglo de vivienda social (19032003), Madrid, Ministerio de Fomento, Ayuntamiento de Madrid et Consejo Económico y Social, 2 vol.

José Ramón Sánchez Domingo (1993) : Apuntes para la historia de Las Rozas, Concejalía de Cultura del Ayuntamiento de Las Rozas, Ediciones La Librería. 
José María Sánchez Ventura (1948) : El problema de la vivienda barata, Zaragoza, Talleres Editoriales "El Noticiero".

María Alexia Sanz Hernández (2000) : Ojos Negros : La memoria de un pueblo, Teruel, Instituto de Estudios Turolenses et Ayuntamiento de Ojos Negros.

Rosa Serra I Rotés (2000) : Les colònies tèxtils a Catalunya, Manresa, Angle Editorial.

Julián Sobrino Simal (1996) : Arquitectura industrial en España, Madrid, Ed. Cátedra.

- (2001) : «El complejo minero de Villanueva del Río y Minas », El Patrimonio Industrial de Andalucía. Jornadas Europeas de Patrimonio 2001, Sevilla, Separata, Gabinete Pedagógico de Bellas Artes.

Tim Strangleman (2002) : «Constructing the past : railway history from below or a study in nostalgia? », The Journal of Transport History, Third series, Vol. 23, nº 2 (septembre 2002), p. 147-158.

Lucy Taksa (2002) : «Mobilisation and spatial resistance : considering the Politics of Location at Sydney's Eveleigh Railway Workshops between the 1920s and the 1960 », in Christian Chevandier et Philippe Mioche (sous la dir. de), "Ateliers et dépôts du Matériel ferroviaire : deux siècles d'histoire ", Actes du 10 colloque de l'AHICF, Arles, 2527 avril 2002, Revue d'histoire des chemins de fer, 28-29 (2003), p. 438-460.

Mercè Tatjer Mir (1998) : «Solucions a la crisi de l'habitatge popular a Barcelona: les primeres experiencies a Sant Andreu (1909-1937)», in Checa, M. (sous la dir. de.), Sant Andreu, de poble a ciutat (1875-1936), p. 61-76.

Eloy Tejada Herrero et Antonio Zavala (1999) : Castejón y el ferrocarril (años 1920 a 1931), Guipúzcoa, Editorial Sendoa.

Luis Juan Tomás García (1991) : La minería sevillana del carbón. Minas de la Reunión y la Compañia de los Ferrocarriles de $M Z A$, Sevillla, Diputación Provincial de Sevilla.

Gabriel Tortella Casares (1973): Los orígenes del capitalismo en España. Banca, industria y ferrocarril en el siglo XIX, Madrid, Tecnos.

Stéphane Vilaire (1999) : «La réhabilitation du site ferroviaire d'HirsonBuire », in « Le patrimoine ferroviaire : enjeux, bilans et perspectives », Actes du 6e colloque de l'AHICF, Mulhouse, 23-26 septembre 1998, Revue d'histoire des chemins de fer, 20-21 (1999), p. 179-190.

Alfredo Villanueva Paredes et Jesús Leal Maldonado (1990) : Historia y Evolución de la Colonización Agraria en España. Volumen III : Laplanificación del regadio y los pueblos de colonización, Madrid, Ministerio de Agricultura, Pesca y Alimentación, Ministerio para las Administraciones Públicas y Ministerio de Obras Públicas y Transportes. 
Francisco Wais Sanmartín (1974) : Historia de los ferrocarriles españoles, Madrid, Editora Nacional.

Luis Zurdo Olivares (1911): Veinte años de vida ferroviaria, Barcelona, Impr. Henrich y cía. 